\title{
A2A adenosine receptor modulates drug efflux transporter P-glycoprotein at the blood-brain barrier
}

\author{
Do-Geun Kim and Margaret S. Bynoe \\ Department of Microbiology and Immunology, College of Veterinary Medicine, Cornell University, Ithaca, New York, USA.
}

\begin{abstract}
The blood-brain barrier (BBB) protects the brain from toxic substances within the peripheral circulation. It maintains brain homeostasis and is a hurdle for drug delivery to the CNS to treat neurodegenerative diseases, including Alzheimer's disease and brain tumors. The drug efflux transporter P-glycoprotein (P-gp) is highly expressed on brain endothelial cells and blocks the entry of most drugs delivered to the brain. Here, we show that activation of the A2A adenosine receptor (AR) with an FDA-approved A2A AR agonist (Lexiscan) rapidly and potently decreased P-gp expression and function in a timedependent and reversible manner. We demonstrate that downmodulation of P-gp expression and function coincided with chemotherapeutic drug accumulation in brains of WT mice and in primary mouse and human brain endothelial cells, which serve as in vitro BBB models. Lexiscan also potently downregulated the expression of BCRP1, an efflux transporter that is highly expressed in the CNS vasculature and other tissues. Finally, we determined that multiple pathways, including MMP9 cleavage and ubiquitinylation, mediated P-gp downmodulation. Based on these data, we propose that A2A AR activation on BBB endothelial cells offers a therapeutic window that can be fine-tuned for drug delivery to the brain and has potential as a CNS drug-delivery technology.
\end{abstract}

\section{Introduction}

The brain is one of the most vascularized organs in the body. This high vascularity enables the efficient and constant supply of oxygen and nutrients from the peripheral circulation to the brain to maintain its proper function $(1,2)$. The brain vasculature is lined by a single layer of specialized endothelial cells that provide a physical barrier against entry of unwanted substances from the circulation (3). In addition, tight and adherens junction molecules seal the spaces between adjacent endothelial cells, generating even greater resistance (4). This physical separation by endothelial cells and junction molecules forms the blood-brain barrier (BBB). Also, the BBB is insulated with extracellular matrix proteins, pericytes, and astrocytic end-feet processes, creating stability, insulation, and extremely high resistance. Together, these are referred to as the neurovascular unit (NVU) $(2,5)$. In addition to providing a physical barrier, brain endothelial cells are equipped with efflux and influx transporters and receptors. These influx and efflux proteins are also expressed on astrocytic end-feet processes and thus selectively regulate the entry of substances into the brain (5). The high resistance of the $\mathrm{BBB}$ does not allow molecules larger than $450 \mathrm{Da}$ to cross the $\mathrm{BBB}$. This is critical to limiting entry of harmful substances, including infectious agents and toxins, into the brain and to maintaining the complex brain physiology and strict ionic environment $(2,6)$.

However, while the protection provided by the BBB is essential to the health of the host, it hampers the delivery of drugs into the brain to treat neurological disorders such as Alzheimer's disease (AD) or primary brain cancers $(3,4)$. Many available drugs with

Conflict of interest: The authors have declared that no conflict of interest exists. Submitted: September 21, 2015; Accepted: February 18, 2016.

Reference information: J Clin Invest. 2016;126(5):1717-1733. doi:10.1172/JCI76207. the potential to treat these diseases are not effectively delivered to the brain due to the physical hindrance and efflux transporters imposed by the BBB. There have been numerous attempts to overcome the hindrance of drug delivery by the BBB that include physical disruption of the $\mathrm{BBB}$, drug modification for easier passage across the $\mathrm{BBB}$, and intrathecal injection of drugs into the brain (7-10). However, these approaches have suffered from shortcomings, including toxicity, decreased drug efficacy, and invasiveness, that can result in permanent brain damage.

Cells and soluble factors cross the BBB through the paracellular or transcellular pathways $(11,12)$. Passage across the paracellular pathway disrupts cell-to-cell junction to permit access to the brain. On the other hand, the transcellular pathway is mediated through transporters highly expressed on the luminal side of brain endothelial cells that allow for selective entry of molecules into the brain while maintaining normal brain physiology $(12,13)$. However, multidrug-resistant (MDR) transporters, especially drug efflux transporters, are highly expressed on brain endothelial cells and hinder the effective delivery of drugs into the CNS, including P-glycoprotein (P-gp) and breast cancer-resistant protein 1 (BCRP1/ ABCG2) $(3,9,14)$. The most widely known and studied drug transporter expressed on brain endothelial cells is P-gp. $(15,16)$. P-gp was the first MDR human transporter to be identified. It was first observed and described in drug-resistant cancer cells that highly expressed it. In breast cancer cells, P-gp prevents effective chemotherapeutic treatment by blocking chemotherapeutic drug uptake (17-19). Later studies showed that P-gp is also highly expressed on capillaries of the liver, sex organs, and the brain and is involved in expulsion of xenobiotics from the CNS (20-23). P-gp is composed of 2 ATP-binding cassettes (ABC) and 2 transmembrane domains. The drug-binding pocket of $\mathrm{P}$-gp is nonspecific, and this allows for a broad spectrum of drugs as substrates (24-26). Recent studies sug- 
gest that substrates that are bound to the binding pocket of P-gp and are expelled into the extracellular space undergo conformational changes upon consumption of ATP as an energy source (26). Inhibitors of P-gp are widely used in preclinical and clinical studies for overcoming MDR. Valspodar (PSC833) is a functional inhibitor that binds directly to the drug-binding pocket of P-gp, thereby allowing entry of P-gp substrates into the cell $(27,28)$. Previous studies also proposed a possible role for P-gp in processing of intracellular cholesterol and transport of cytokines, steroid metabolites, and lipids to the extracellular space $(24,29,30)$. P-gp is mostly localized on cell membranes in drug-resistant cancer cells, whereas it is mostly localized in the cytoplasm, including Golgi apparatus, lysosome, mitochondria, and endosomes, in non-drug-resistant cancer cells (31-34). It is also suggested that P-gp actively circulates between organelles in the cell via the endocytic pathway (34). BCRP1 is another MDR transporter with an $\mathrm{ABC}$ that is expressed in the gut, $\mathrm{BBB}$, placenta, and testis. Its expression in cancer cells indicates poor prognosis, which is believed to be caused by its regulation by multiple mechanisms of drug resistance (35).

In brain endothelial cells, P-gp is localized in lipid raft, more specifically, in caveolae. Caveolae are specialized membrane (lipid) rafts that contain the caveolin protein and are characterized as flask-like invaginations of the plasma membrane. Caveolaemediated endocytosis has been shown to facilitate the transport of molecules to other parts of the cell (36). An important characteristic of caveolae is detergent insolubility, which can be exploited for fractionation and enrichment (37-40). However, whether P-gp constantly circulates in cells and is recruited to the cell membrane upon its encounter with its substrate in the cytoplasm or persists on the cell membrane and pumps out its substrates into the extracellular space is not clearly proven. In a recent report, mitomycin $\mathrm{C}$ treatment induced the translocation of the cytoplasmic portion of P-gp to the cell surface, suggesting that relocalization of P-gp can be induced by chemical triggers (41). Many drugs developed for treatment of brain disorders are largely classified as P-gp substrates; thus, significant efforts are placed on developing methods to bypass the hindrance posed by P-gp $(27,42,43)$. Recent studies from our lab showed adenosine receptor (AR) signaling regulates the permeability of the $\mathrm{BBB}(44,45)$.

Adenosine is a purine nucleoside that functions as an important local signaling molecule and is involved in various physiological functions, including neurotransmission, cardiac pace, and immune regulation (46-48). Extracellular adenosine is generated by conversion of ATP into ADP and AMP by nucleoside triphosphate diphosphohydrolase-1 (CD39), and AMP is further converted to adenosine by the action of $5^{\prime}$-ectonucleotidase (CD73). Extracellular adenosine mediates its action through its 4 receptors, A1, A2a, A2b, and A3. ARs are 7-transmembrane G protein-coupled receptors that are widely distributed on various cell types in the body, and their activation induces changes in the level of second messenger signaling, including $\mathrm{Ca}^{2+}$ or cAMP (48-50). In our previous study, we showed that mice lacking CD73 and unable to synthesize extracellular adenosine have tighter BBBs and are protected from experimental autoimmune encephalomyelitis (EAE), the animal model for multiple sclerosis $(51,52)$. More recently, we showed that activation of A2A AR with a broad-spectrum AR agonist or an FDA-approved specific A2A AR agonist,
2-\{4-[(methylamino)carbonyl]-1H-pyrazol-1-yl $\}$ adenosine (Lexiscan), increased accumulation of drugs into the brain in a time- and dose-dependent manner (45). BBB opening under AR signaling was reversible. We showed that the duration of $\mathrm{BBB}$ permeability was dependent on the half-life of the AR agonist. In our recent work, we showed that activation of AR signaling exerted its effects on the paracellular pathway by altering VE-cadherin and claudin- 5 expression to promote BBB permeability in human primary brain endothelial cells. We demonstrated that activation of AR signaling with Lexiscan or 5'-N-ethylcarboxamidoadenosine (NECA) mediated BBB permeability by Rho-GTPase modulation (44).

In this study, we set out to determine whether activation of A2A AR signaling exerts its effects on the transcellular pathway by way of P-gp modulation. To test this, we used the FDA-approved A2A AR agonist Lexiscan or the broad-spectrum AR agonist NECA to determine the impact of A2A AR activation on P-gp expression and function. We observed that activation of the A2A AR by Lexiscan or NECA downmodulated the expression level of P-gp in primary mouse and human brain endothelial cells. P-gp expression was downmodulated by A2A AR signaling, and this downmodulation coincided with increased accumulation of Rhodamine 123 (Rho123), a classical P-gp substrate. Further, we observed that A2A AR activation by Lexiscan rapidly increased accumulation of the chemotherapeutic drug and P-gp substrate epirubicin in the mouse brain. NECA also increased the accumulation of epirubicin at later time points compared with Lexiscan. Similar to P-gp, we found that Lexiscan potently downmodulated the expression of BCRP1, whereas NECA's effect was less potent. These data strongly suggest that A2A AR signaling potently increases the transcellular permeability of BBB endothelial cells. We anticipate that, in the future, these studies could have a high impact on the field of drug delivery to the brain to treat neurodegenerative diseases.

\section{Results}

$P$-gp is highly expressed in primary human brain endothelial cells and a human brain endothelial cell line. Studies have shown that P-gp is highly expressed on the luminal side of BBB endothelial cells and on the plasma membrane due to its functional property as a transporter (16). First, to confirm expression of P-gp in human brain endothelial cells, we performed immunofluorescence assay (IFA) of endothelial cells with an antibody specific for P-gp. We observed that HCMEC-D3 cells, a human brain endothelial cell line, expressed abundant P-gp in the cytoplasm and, to a lesser extent, on the cell surface (Figure 1A). Similarly, in human primary brain endothelial cells, HBMVEC, we observed high P-gp expression (Figure 1A). This mostly cytoplasmic P-gp localization in brain endothelial cells is reminiscent of a P-gp expression pattern previously reported in human primary brain endothelial cells (53). However, this expression pattern of P-gp localization is different from the expression pattern observed in drug-resistant cancer cells, which showed mostly surface expression (31). Thus, it is possible that the location/localization of P-gp is different in transformed versus normal cells and/or is different in different cell types.

In previous studies, it was reported that P-gp in brain endothelial cells is strongly colocalized with caveolae, which are specialized microdomains of cell membranes that can be separated 
A
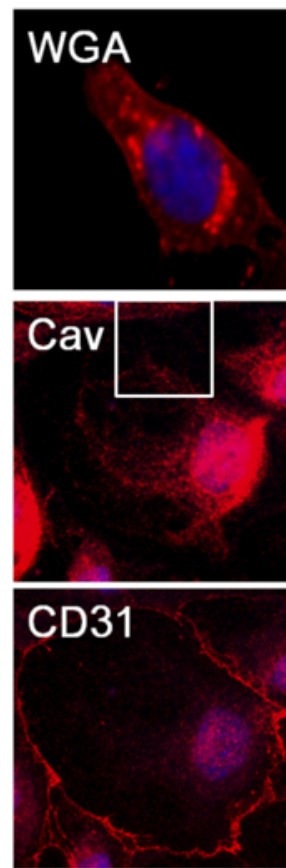

D

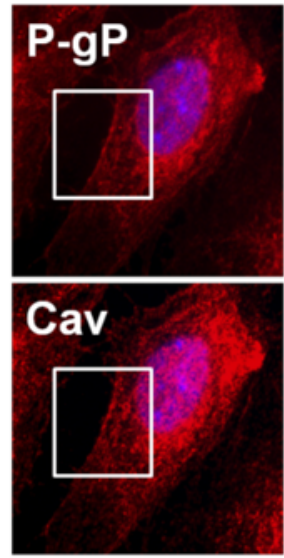

F

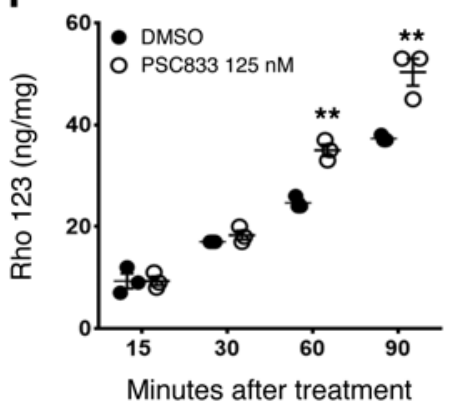

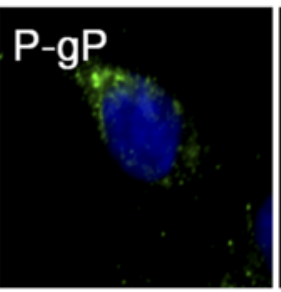
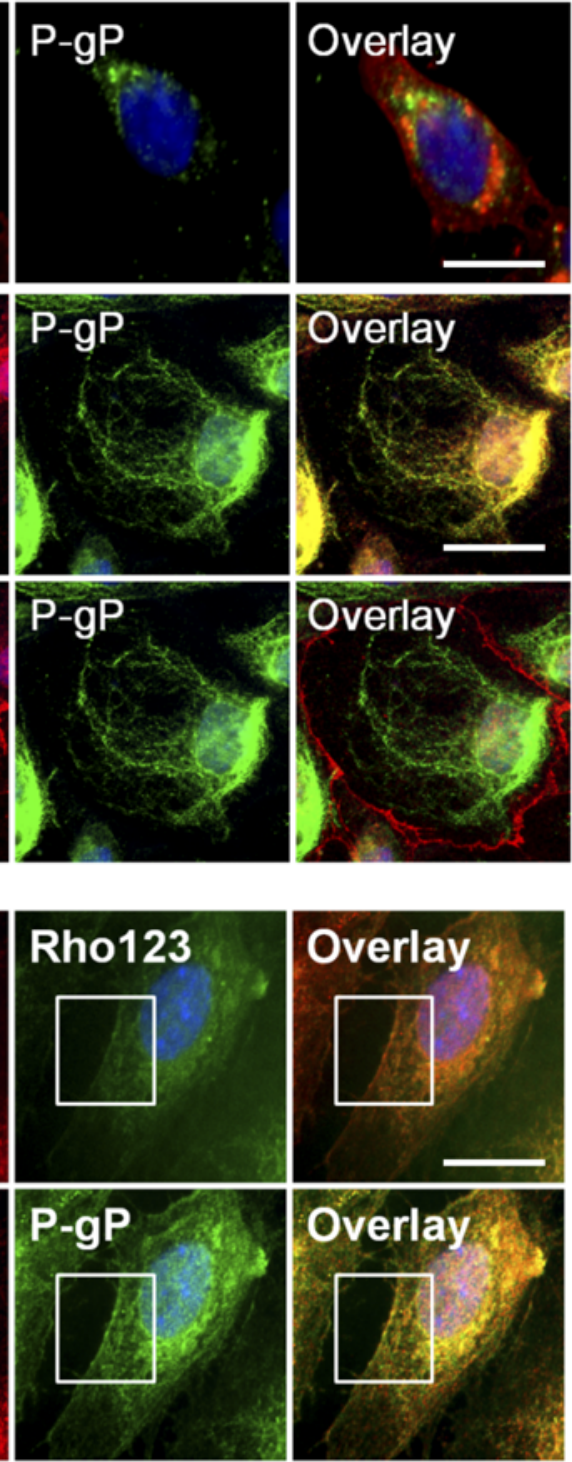

G

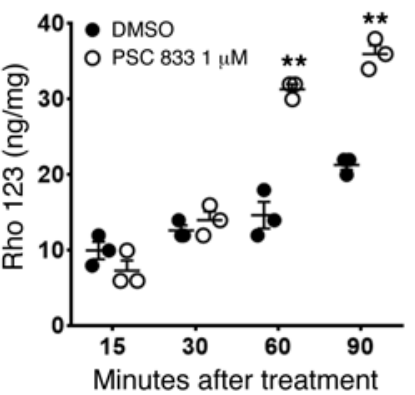

B
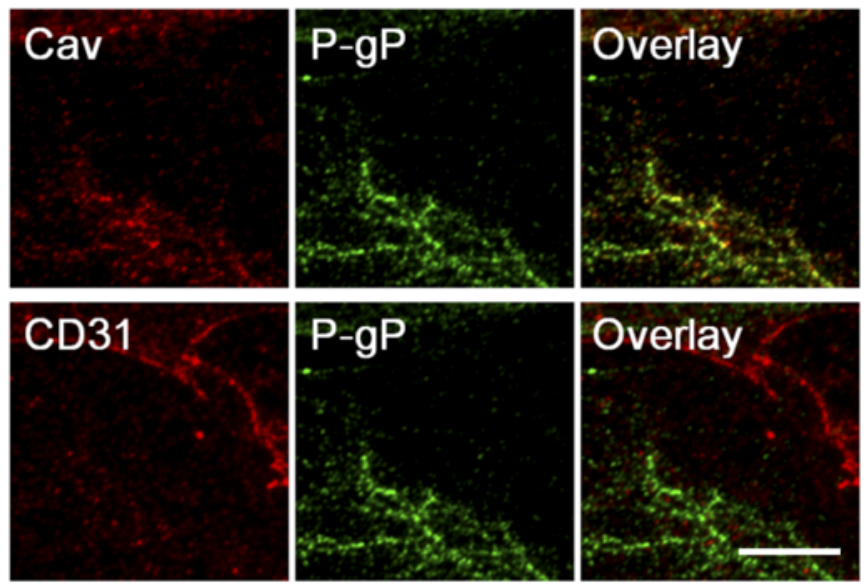

C

Input:P-gP

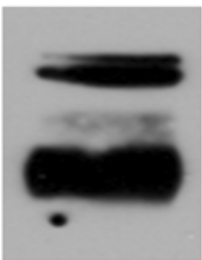

$\mathbf{E}$

IB:Caveolin
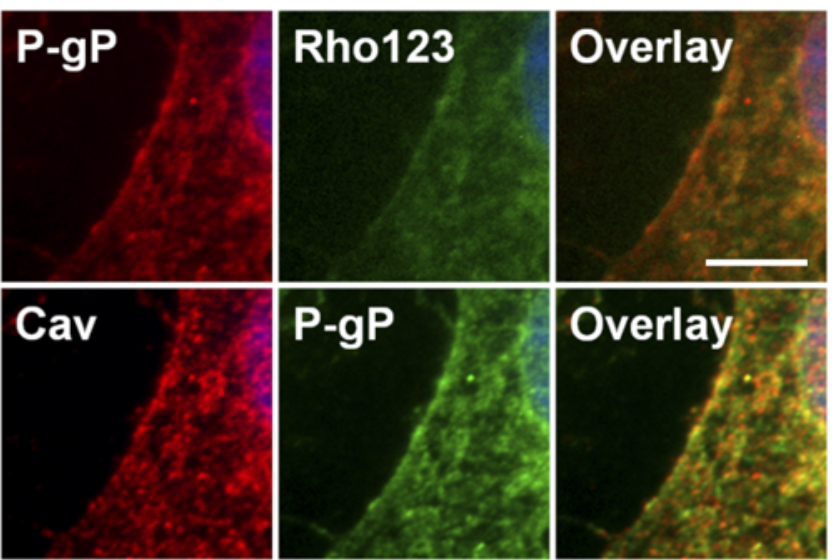

Figure 1. P-gp is expressed mostly in the cytoplasm of human brain endothelial cells and is colocalized with caveolae. (A) P-gp (green) is expressed in the cytoplasm of a human brain endothelial cell line, HCMEC-D3 (top panels), and in human primary brain endothelial cell, HBMVEC (middle and bottom panels). HCMEC-D3 (top panels) were counterstained with WCA (red) to depict cell membrane. HBMVEC were stained with an antibody to caveolin-1 (middle panels) (red) or antibody to CD31 (bottom panels) (red) as markers for caveolae and cell membrane, respectively. Nucleus was counterstained with DAPI (blue). Scale bars: $25 \mu \mathrm{m}$. (B) Representative enlarged images from inset in $\mathbf{A}$ of HBMVEC cells. Scale bar: $10 \mu \mathrm{m}$. (C) P-gp was pulled down (immunoprecipitated) using anti-P-gp antibody and immunoblotted (IB) with an anticaveolin-1 antibody. (D) HBMVEC was treated with Rho123 (green, top panel) for 1 hour, then fixed and stained with anti-P-gp (red in top panel or green in bottom panel).Caveolae were counterstained with anti-caveolin-1 (green, bottom panel). Scale bar: $25 \mu \mathrm{m}$. (E) Enlarged images from inset in $\mathbf{D}$ depicting P-gp or caveolae colocalization. Scale bar: $10 \mu \mathrm{m}$. (F and $\mathbf{G}$ ) Inhibition of P-gp with PSC833, a functional inhibitor of P-gp in Rho123 uptake in human brain endothelial cell line HCMEC-D3 (F) and primary human brain endothelial cells (C). ${ }^{* *} P<0.01$ ( $n=3$, 2-tailed Student's $t$ test, 1 representative result from 3 different experiments). 

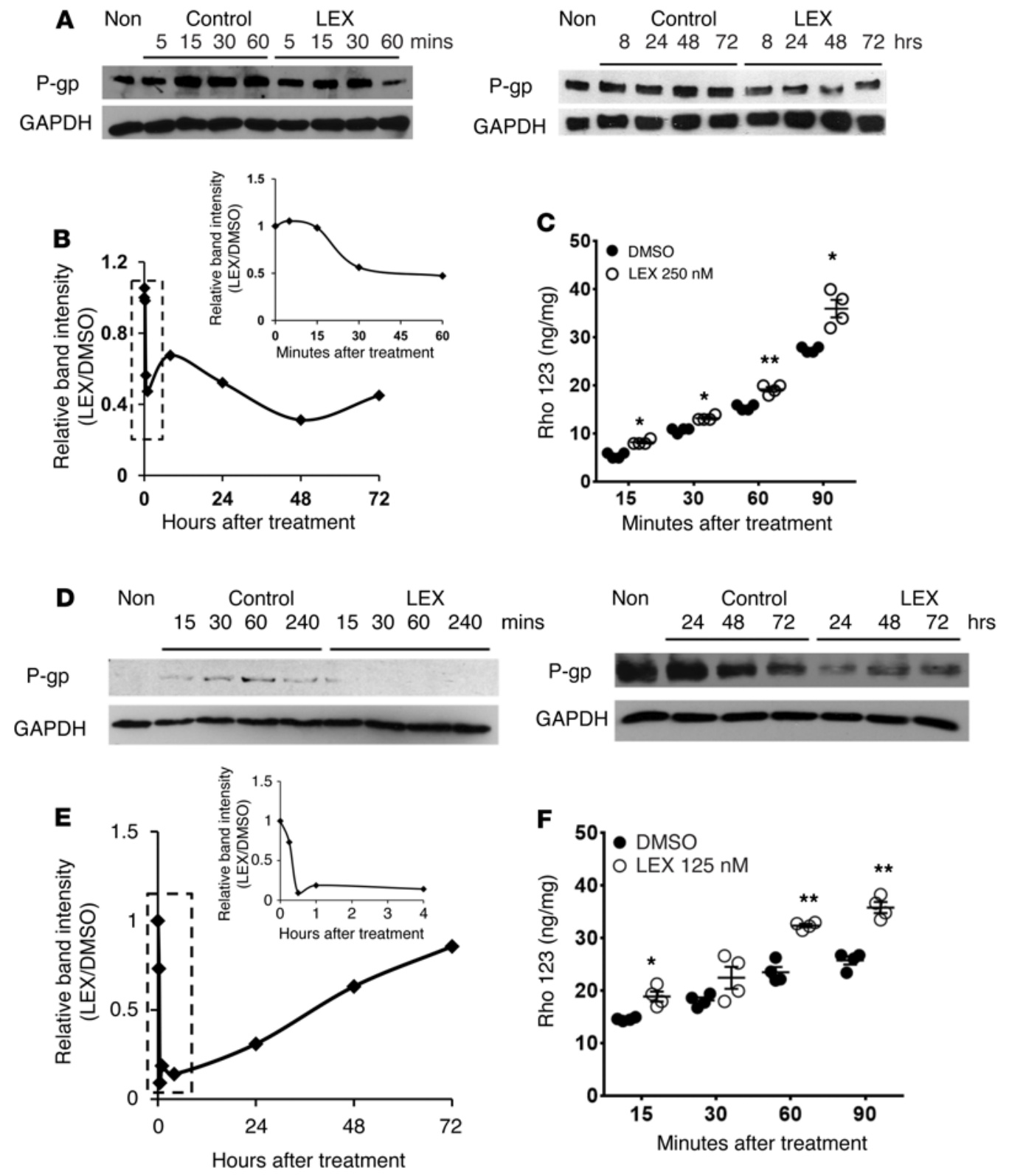

G
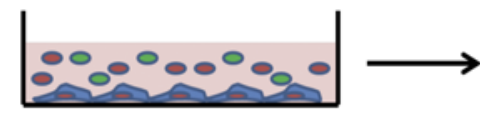

Cells were lysed for

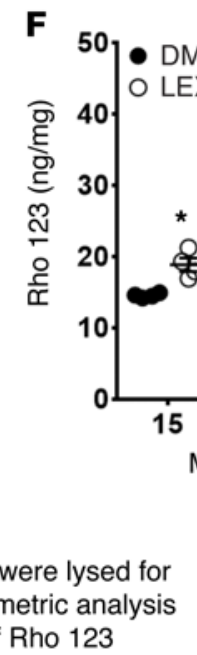

- Rho 123 (ng/mg)

- Lexiscan or NECA

Human brain endothelial cell
Figure 2. Activation of A2A AR downmodulates P-gp expression and function in human brain endothelial cells. (A) Western blot analysis of P-gp expression in HCMEC-D3 cells treated with Lexiscan (LEX) $(1 \mu \mathrm{M})$ for up to 72 hours. GAPDH was used as loading control. (B) For densitometric analysis, the intensity of P-gp upon Lexiscan treatment was divided by that of DMSO control and graphed. Densitometric analysis of short-time point (dotted box) is depicted as an inset. (C) Rho123 uptake assay of HCMEC-D3 cells treated with Lexiscan $(1 \mu \mathrm{M})$ was performed. Concentrations of Rho123 accumulation in brain endothelial cells were analyzed by fluorometry (Synergy, Biotek), with excitation at $488 \mathrm{~nm}$ and emission at $523 \mathrm{~nm}$. ${ }^{*} P<$ 0.05 ; ${ }^{*} P<0.01(n=4,2$-tailed Student's $t$ test, 1 representative result of 3 different experiments). (D) Western blot analysis depicting P-gp expression in human primary brain endothelial cells treated with Lexiscan (1 $\mu \mathrm{M})$ for up to 72 hours. GAPDH was used as loading control. (E) Densitometric analysis was used to measure the intensity of $\mathrm{P}$-gp expression with Lexiscan or NECA treatment and was divided by the DMSO control and graphed. Densitometric analysis of short-time point (dotted box) is depicted as an inset. (F) Rho123 uptake assay of human primary brain endothelial cells treated with Lexiscan $(1 \mu \mathrm{M})$. The concentration of Rho123 accumulation in endothelial cells was quantified by fluorometry (Synergy, Biotek), with excitation at $488 \mathrm{~nm}$ and emission at 523 nm. ${ }^{*} P<0.05 ;{ }^{* *} P<0.01$ ( $n=4$, 2-tailed Student's $t$ test, 1 representative result of 3 different experiments). (C) Schematic diagram of Rho123 accumulation assay from $\mathbf{C}$ and $\mathbf{F}$. by cell fractionation assay $(16,37,54)$. To determine whether P-gp is associated with caveolae, we costained P-gp and caveolin-1, which is a major caveolae-associated protein $(37,54)$. In human primary brain endothelial cells, we observed strong colocalization of P-gp with caveolin-1 (Figure 1, A and B). To determine whether P-gp colocalizes with caveolin-1, we performed immunoprecipitation assay using P-gp antibody and observed strong interaction between P-gp and caveolin-1 (Figure 1C). These results are in line with previous studies and suggest that caveolin-1 is involved in P-gp trafficking between the cell membrane and other cell compartments $(54,55)$. Interestingly, we also observed colocalization of P-gp with caveolae mostly in the cytoplasm rather than on the cell surface in both human and mouse primary brain endothelial cells (Figure 1, A and B, and Supplemental Figure 1, A and B). While previous studies showed P-gp colocalized with caveolae, this is the first study, to our knowledge, that shows that P-gp colocalization 
with caveolae occurs in the cytoplasm. Recent studies showed that caveolin- 1 is incorporated in the endosome called a cavicle, which is distinct from classical endosomes, and this special endosome actively delivers target proteins (including glycosylphosphatidylinositol-anchored [GPI-anchored] proteins) to the cell membrane $(38,56,57)$. To determine whether P-gp actively circulates by caveolae transport to relocate to the site where substrate is present and ultimately to the cell surface, we treated primary human brain endothelial cells with the P-gp substrate Rho123 for 1 hour. We observed strong colocalization of P-gp with Rho123 and surface localization of P-gp (Figure 1, D and E). Also, P-gp was strongly colocalized with caveolin-1 both on the cell surface and in the cytoplasm (Figure 1, D and E). These observations strongly suggest that P-gp may actively circulate in these endothelial cells by the caveolae-protein transport system and that upon stimulation (trigger) by its substrate, it relocalizes to the substrate's site. Thus, the cytoplasmic portion of P-gp appears to bind to its substrate and deliver it to the cell surface for expulsion.

Since we observed mostly cytoplasmic localization of P-gp (in contrast with cell-surface localization observed in MDR cancer cells) (34), we next determined P-gp functionality using Rho123 uptake assay. Rho123 (a substrate of P-gp) is widely used to measure P-gp function, since its intracellular accumulation has a reciprocal relationship to P-gp levels (58). Brain endothelial cells were treated with a competitive inhibitor of P-gp, PSC833, and Rho123 uptake was measured. PSC833 is a functional inhibitor that directly binds to the drug-binding pocket of P-gp, thereby allowing entry of P-gp substrate into the cell. We observed that Rho123 accumulation increased in a time-dependent manner in both human primary brain endothelial cells and the human brain endothelial cell line (Figure 1, F and G). P-gp downmodulation was observed beginning at 60 minutes and was maintained up to 90 minutes, indicating that P-gp function was effectively downmodulated in HCMEC-D3 cells by the functional inhibitor (Figure $1 \mathrm{~F}$ ). P-gp downmodulation was observed in primary human brain endothelial cells beginning at 60 minutes and was maintained up to 90 minutes (Figure 1G). These results indicate that these cells are valid in in vitro models to test the modulation and function of P-gp by AR signaling.

Activation of A2A AR downmodulates $P$ - $g p$ expression and function in brain endothelial cells. Previously, we have shown that ARmediated signaling increases the permeability of the BBB to large molecules in vivo and in vitro $(44,59)$. In this study, to test the hypothesis that AR signaling regulates P-gp function, we used in vitro $\mathrm{BBB}$ models to determine whether AR signaling can indeed inhibit the expression and function of P-gp. First, we tested the effect of AR signaling on P-gp expression levels in primary mouse brain endothelial cells that were grown to form monolayers and then treated with or without $1 \mu \mathrm{M}$ of the A2A AR agonist Lexiscan or NECA, a broad-spectrum AR agonist, at different time points up to 72 hours. In Lexiscan-treated samples, Western blot analysis showed rapid downmodulation of P-gp beginning at $30 \mathrm{~min}$ utes, which was maintained for up to 1 hour (Supplemental Figure 2 , A and B). This was reversed at 4 hours and began to decrease thereafter up to 48 hours, then reversed at 72 hours (Supplemental Figure 2, A and B). In NECA-treated primary (mouse) brain endothelial cells, P-gp expression decreased very rapidly and remained downmodulated for up to 4 hours; by 8 hours, it returned to baseline levels (Supplemental Figure 2, C and D).

To verify the "visual" presence of the P-gp substrate and to map its timing and localization in endothelial cells, we next performed similar experiments in HCMEC-D3 and in human primary brain endothelial cells by IFA. Monolayers of HCMEC-D3 cells were treated with Lexiscan or NECA, and P-gp expression was analyzed by Western blot analysis. In HCMEC-D3 cells, Lexiscan treatment induced a rapid decrease in P-gp expression beginning at 30 minutes, which was maintained for up to 1 hour (Figure 2, A and B). Interestingly, at 24 to 48 hours, P-gp expression decreased even more prominently (Figure 2, A and B). NECA also decreased P-gp expression for up to 4 hours, and P-gp returned to basal levels at 8 hours and declined again between 48 and 72 hours (Supplemental Figure 3, A and B). As proof that P-gp downmodulation by Lexiscan and NECA correlates with increased substrate accumulation, we evaluated accumulation of Rho123 in brain endothelial cells. Lexiscan increased Rho123 accumulation in the HCMEC-D3 cell line beginning at 15 minutes, and this was maintained for up to 90 minutes (Figure 2C). NECA treatment showed a similar trend in Rho123 accumulation, although it was not statistically significant (Supplemental Figure 3C). This suggests that a decrease in P-gp expression level by AR activation is consistent with decreased P-gp functionality.

As cell lines do not always reproduce all the characteristics of primary cells, we next examined the effect of Lexiscan and NECA treatment on P-gp expression in primary human brain endothelial cells. Both Lexiscan and NECA treatment exerted effects on P-gp expression in human primary brain endothelial cells similar to those observed in the human brain endothelial cell line HCMEC-D3 (Figure 2D and Supplemental Figure 3D). Lexiscan induced rapid downmodulation of P-gp expression, which began to decrease at 15 minutes and was maintained at a decreased level for up to 4 hours (Figure 2, D and E). This downmodulatory trend was once again induced for up to 48 hours and was recovered by 72 hours (Figure 2, D and E). In the NECA-treatment group, the expression level of P-gp began to decrease at 30 minutes and was maintained for up to 48 hours and recovered by 72 hours (Supplemental Figure 3, D and E). This was recapitulated in the Rho123 uptake assay, which showed that Lexiscan rapidly suppressed P-gp function beginning at 15 minutes and that this was maintained for up to 90 minutes (Figure 2F). NECA showed a trend in P-gp suppression, but it did not reach statistical significance (Supplemental Figure 3F). We next determined whether P-gp downregulation results in substrate accumulation in a transmigration assay using primary human brain endothelial cells (experimental procedure described in Figure 3A). Lexiscan induced rapid increase in permeability to Rho123 beginning at 5 minutes, and this lasted for up to 60 minutes (Figure 3B). Rho123 levels returned to steady state by 2 hours; they increased again by 4 hours, and this was maintained at steady state for up to 12 hours (Supplemental Figure 4). NECA's effect on Rho123 levels occurred at 12 hours and was maintained for up to 24 hours (Figure 3B and Supplemental Figure 4). To further dissect the effect of AR signaling on the functionality of P-gp and Rho123 accumulation in primary human brain endothelial cells, endothelial cells were treated with Rho123 with or without Lexiscan, and Rho123 accumulation was 
A

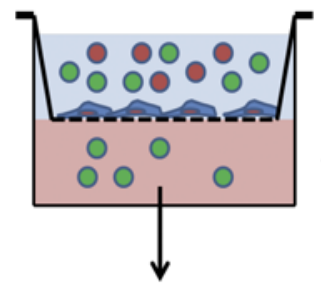

Media was collected for

fluormetric analysis

at different time point
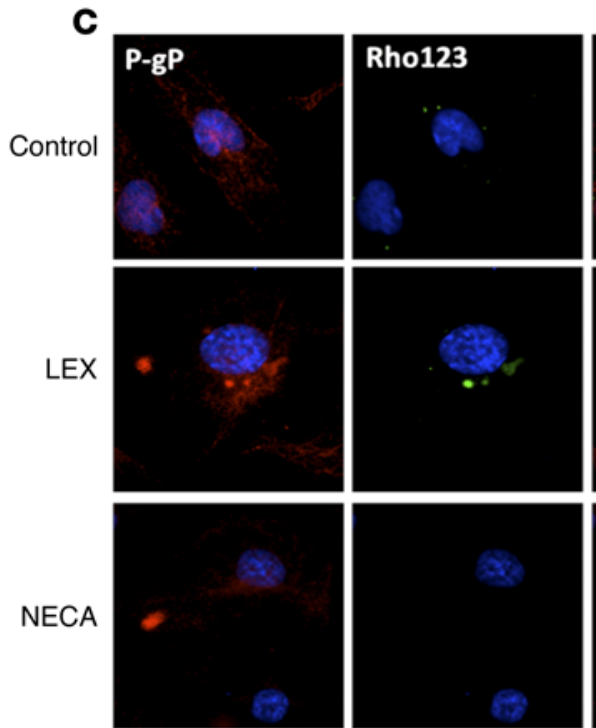

$15 \min$
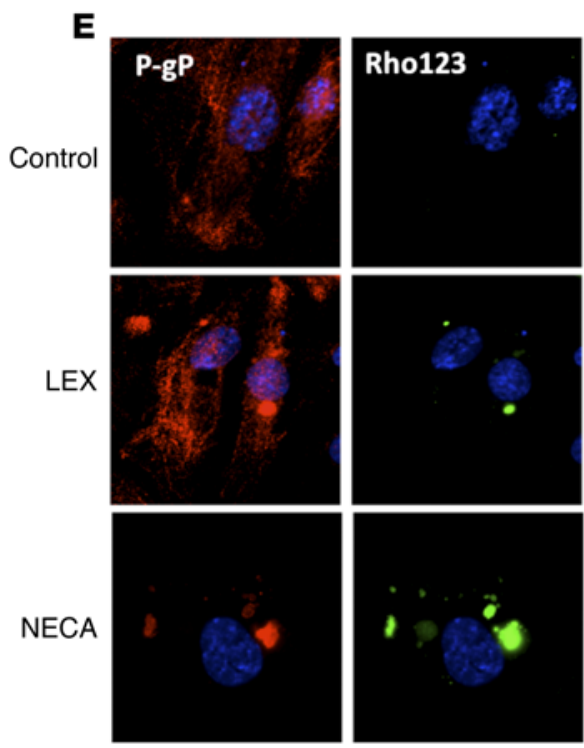

4 hour
$\mathbf{B}$
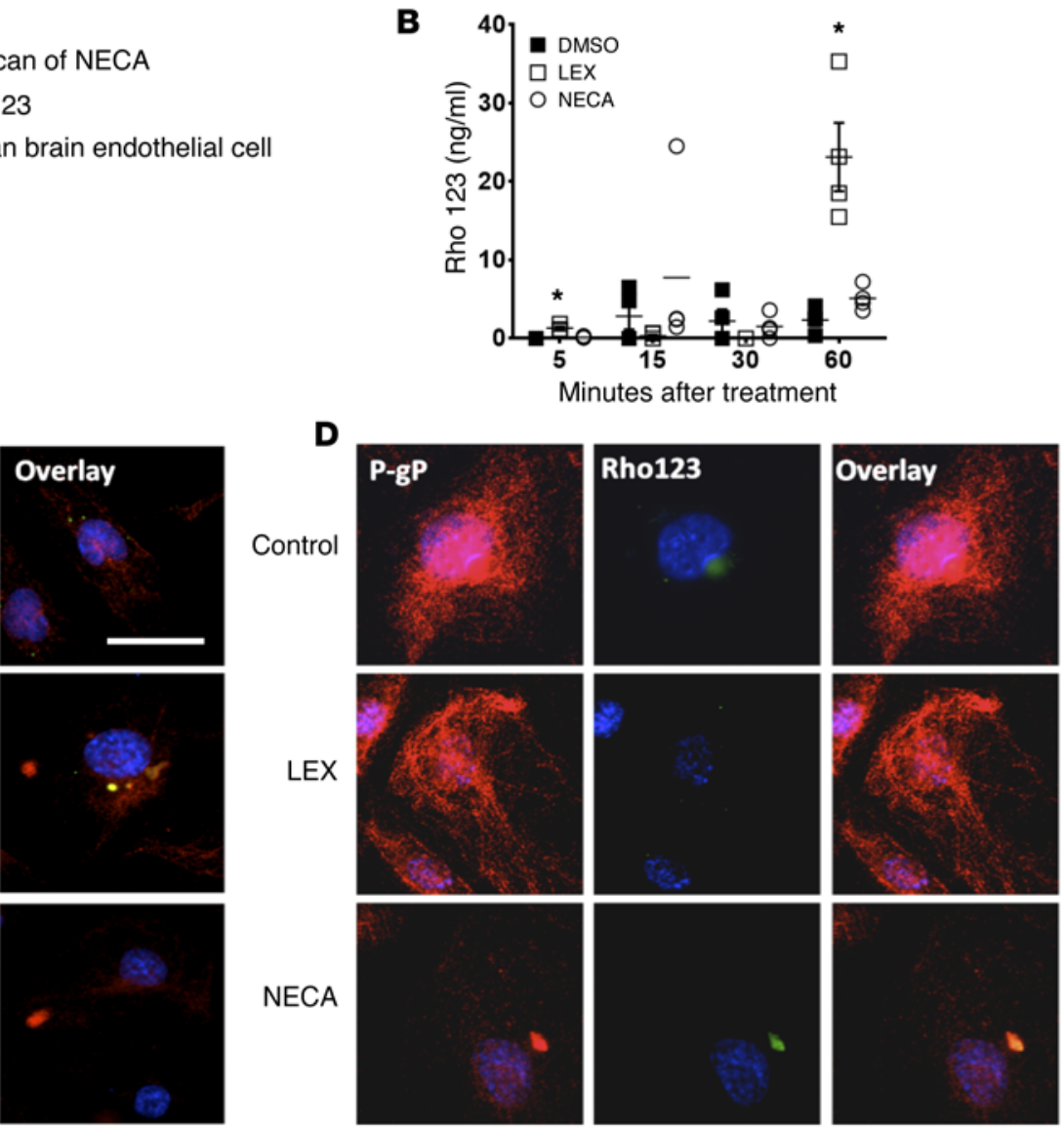

1 hour
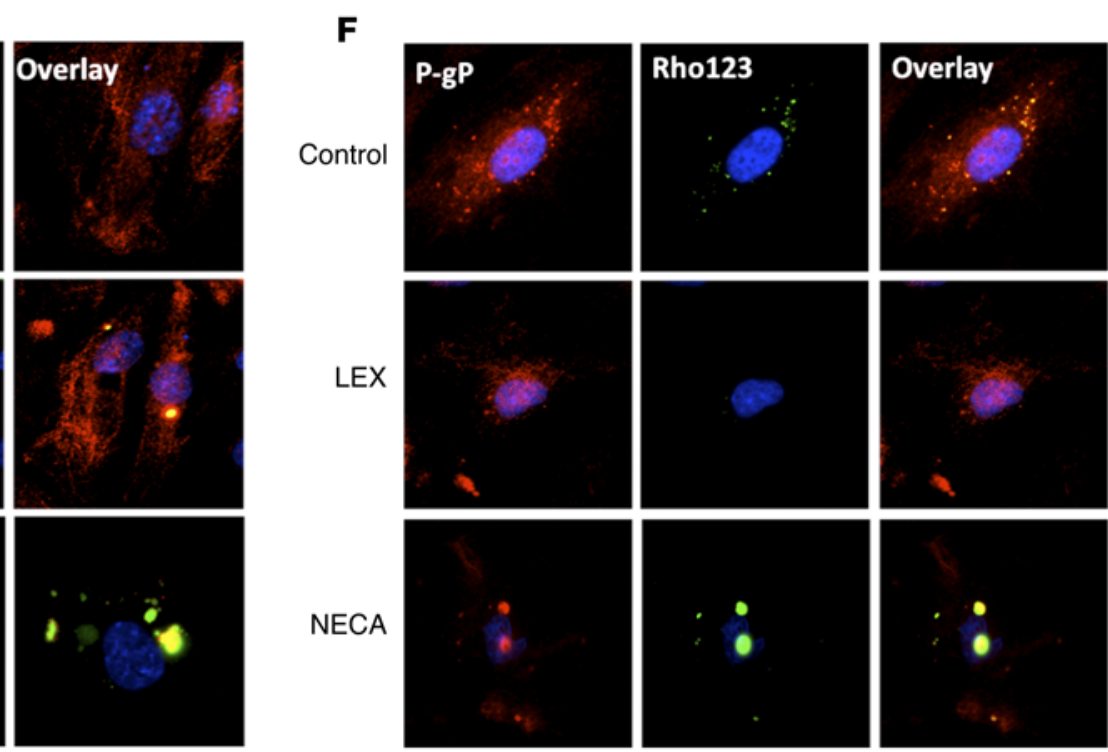

24 hour

Figure 3. Activation of A2A AR by Lexiscan induces rapid transmigration of Rho123 across an in vitro human BBB and accumulation of Rho123 in primary brain endothelial cell. (A) Diagram depicting methodology of transmigration assay of Rho123 using an in vitro BBB model. (B) Results of in vitro BBB model using primary human brain endothelial cell monolayers cultured on porous membranes to determine Rho123 migration across the BBB. Endothelial cell monolayers cultured on porous membranes were treated with Lexiscan $(0.25 \mu \mathrm{M})$ or NECA $(0.25 \mu \mathrm{M})$ concomitantly with $2.5 \mu \mathrm{M}$ of Rho123, and the concentration of Rho123 at the bottom chambers was analyzed by fluorimetry, with excitation at $488 \mathrm{~nm}$ and emission at $523 \mathrm{~nm}$. ${ }^{*} P<0.05$ ( $n=$ 4, 2-tailed Student's $t$ test, 1 representative result of 3 different experiments). (C-F) Human primary brain endothelial cells were cultured on coverslips and treated with $2.5 \mu \mathrm{M}$ of Rho123 with or without $1 \mu \mathrm{M}$ of Lexiscan or NECA at different time points (15 minutes, 1, 4, 24 hours). Cells were fixed with $4 \%$ PFA and costained with P-gp and visualized with fluorescent microscope. Scale bar: $25 \mu \mathrm{m}$. 
visualized by IFA at different time points. Lexiscan increased the accumulation of Rho123 beginning at 15 minutes to 1 hour. NECA treatment increased Rho123 accumulation at later time points, beginning at 4 hours; this was maintained for up to 24 hours (Figure 3, C-F). This indicates that NECA treatment also increased Rho123 accumulation in primary human brain endothelial cells, as shown by IFA. Overall, these results suggest that activation of A2A AR by Lexiscan potently and rapidly increases the transcellular permeability in human brain endothelial cells in a reversible manner. While NECA exhibited similar effects, its permeability kinetics occurred later and its effects were less potent than Lexiscan's. These results indicate that A2A AR activation has a potent effect on P-gp expression and function and that this effect occurs rapidly with Lexiscan treatment and may involve multiple mechanisms of regulation.

P-gp downmodulation by Lexiscan is mediated by activation of MMP9, ubiquitination, and translocation to the cytoskeletal fraction. To better understand the mechanism behind the rapid downmodulation of P-gp observed after Lexiscan treatment, we used CSK buffer, which extracts insoluble cytoskeletal materials to determine whether P-gp is contained in the insoluble fraction, as this might explain its rapid downmodulation upon Lexiscan treatment (experimental procedure described in Figure 4A). Comparison of P-gp expression levels in cytoskeletal fraction to P-gp in whole lysate showed increased P-gp in the cytoskeletal fraction compared with control (Figure 4, B and C). In cytoskeletal fraction, P-gp levels increased at from 5 to 15 minutes, returned to baseline by 30 minutes, and were maintained at baseline level for up to 60 minutes (Figure 4C). In contrast, in whole lysate, P-gp levels declined compared with vehicle control beginning at 5 minutes and were maintained for up to $60 \mathrm{~min}$ utes (Figure 4B). Activation of A2A AR is known to induce the relocalization of targets such as tyrosine receptor kinase $\beta$ to the insoluble fraction $(60,61)$. It is possible that P-gp in the soluble fraction may have been translocated to the cytoskeletal fraction by A2A signaling at an early time point, which may explain the rapid decrease in P-gp levels upon Lexiscan treatment.

We next explored the possibility that MMP9, which can be induced by various ARs and degrades extracellular matrix molecules, may become activated and cleave P-gp, resulting in its rapid decrease upon Lexiscan treatment $(62,63)$. We observed increased expression of MMP9 beginning at 5 minutes that matched the same kinetics of P-gp decrease by Western blot and IFA (Figure 4, $\mathrm{D}, \mathrm{G}$, and $\mathrm{H}$ ), which was also observed in mouse brain endothelial cells (Supplemental Figure 5). Consistent with our hypothesis, we observed an increase in secreted P-gp that was released into the media with kinetics similar to those found in MMP9 expression. This secreted MMP9 was suppressed by an MMP9-specific inhibitor (Figure 4E), suggesting that secretion of $\mathrm{P}$-gp is mediated by MMP9. Immunoprecipitation assay and IFA showed that A2A AR activation by Lexiscan induced rapid interaction between P-gp and MMP9, suggesting possible cleavage of P-gp by MMP9 at early time points (Figure 4, F and G). Interestingly, the colocalization of P-gp with MMP9 at the cell surface was increased compared with its control, suggesting that P-gp cleavage occurred at the cell surface and that the cleaved P-gp is secreted into the extracellular space (Figure $4 \mathrm{G}$ ). This suggests that MMP9 is at least in part responsible for the early and rapid decrease in P-gp levels upon Lexiscan treatment (Figure 4B).

We next determined whether A2A AR activation by Lexiscan can activate ubiquitination of P-gp, which was reported as one of the mechanisms that regulates P-gp expression (64) and thereby results in its rapid decrease. Primary human brain endothelial cells were treated with Lexiscan for 15 minutes; immunoprecipitation of P-gp was performed, and the eluent was immunoblotted against an antibody for ubiquitin. The result showed that treatment of primary human brain endothelial cells with Lexiscan for 15 minutes induced increased ubiquitinylation of P-gp compared with vehicle control (Figure 5A). This was further captured by strong colocalization of P-gp with ubiquitin after 15 minutes of treatment of primary human brain endothelial cells with Lexiscan, further indicating that Lexiscan can induce rapid ubiquitination of P-gp, which helps to explain its rapid downmodulation by Lexiscan stimulation (Figure 5B). Overall, these data suggest that the rapid decrease in P-gp expression by Lexiscan may be mediated by several different pathways operating sequentially, independently, or simultaneously (proposed model, Figure 5C).

Ablation of CD73 or ARs increased P-gp expression and decreased $P$-gp substrate accumulation in the brain. To determine whether our in vitro data can be captured in vivo, we sought to determine whether signaling via the A2A AR regulates P-gp expression and functionality in the mouse brain. First, we examined primary brain endothelial cells from brains of mice with genetic deletion of A1 (Adora1 $\left.1^{-/}\right)$, A2A AR (Adora2a $\left.a^{-/}\right)$, or CD73 $\left(\mathrm{Cd} 73^{-/}\right)$to determine whether P-gp expression is altered in their absence compared with WT mice. We observed increased expression of P-gp in primary brain endothelial cells from A1 KO, A2A KO, or CD73 KO mice compared with WT controls (Figure 6A). Moreover, we observed significant decreases in the accumulation of P-gp substrate Rho123 in primary brain endothelial cells (Figure 6B). We next determined whether increased P-gp is observed in the endothelial cells within the brains of these $\mathrm{KO}$ animals. We found that A2A $\mathrm{AR}$ and CD73 KO animals showed stronger P-gp signals than A1 AR KO mice, which showed P-gp expression similar to that of WT mice. This indicates that extracellular adenosine acting through its $\mathrm{A} 2 \mathrm{~A}$ receptor is the major signaling component involved in P-gp expression/function (Figure 6, C and D).

$A R$ activation downmodulates $P$ - $g$ p expression and function in $W T$ mice. Since we observed increased expression of P-gp in brain capillaries of A2A AR KO mice, we next determined whether activation of A2A AR with Lexiscan or NECA can downregulate P-gp expression with concomitant increase in drug accumulation in the brains of WT mice. Lexiscan treatment decreased P-gp expression in brain endothelial cells in WT mice between 5 and 15 minutes; this was recovered by 30 minutes, as shown by Western blot analysis (Supplemental Figure 6, A-C) and IFA (Figure 7, A and B). To assess whether A2A AR activation may have similar downmodulatory effects on other transporters expressed on brain endothelial cells, we next determined the expression level of BCRP1/ ABCG2, which is another key drug transporter highly expressed in many tissues, including the CNS vasculature, by Western blot. We observed that Lexiscan treatment potently downregulated BCRP expression by 15 minutes, after which its expression began to recover by 60 minutes (Supplemental Figure 
A

- Collection of supernatant (for excreted P-gp)

- Lysed with RIPA buffer (for soluble P-gp)

- Lysed with CSK buffer (for insoluble P-gp)

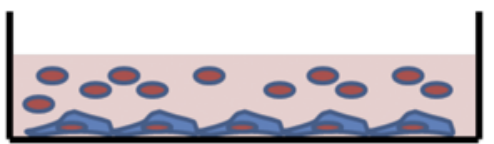

Treated with Lexiscan NECA

- Lexiscan or NECA

$\frown$ Human brain endothelial cell

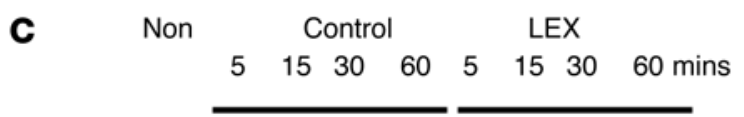

P-gp

GAPDH

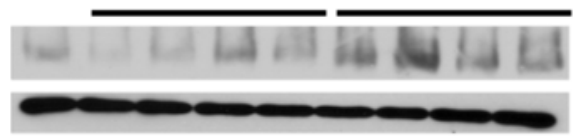

CSK

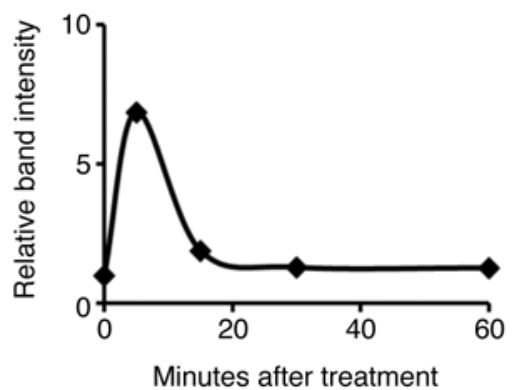

E Non Control LEX

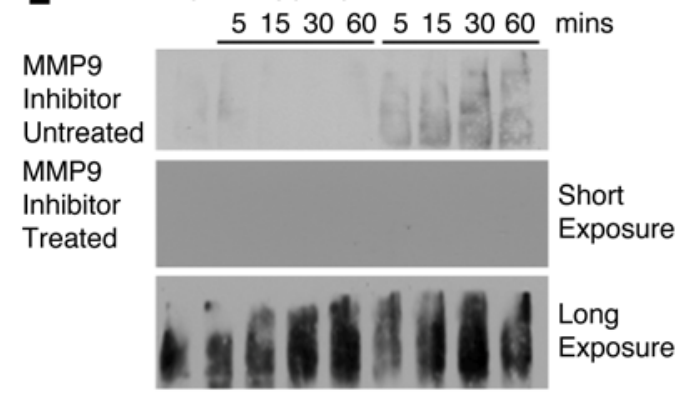

$\mathbf{F}$
B Non Control LEX $\begin{array}{llllllll}5 & 15 & 30 & 60 & 5 & 15 & 30 & 60 \text { mins }\end{array}$
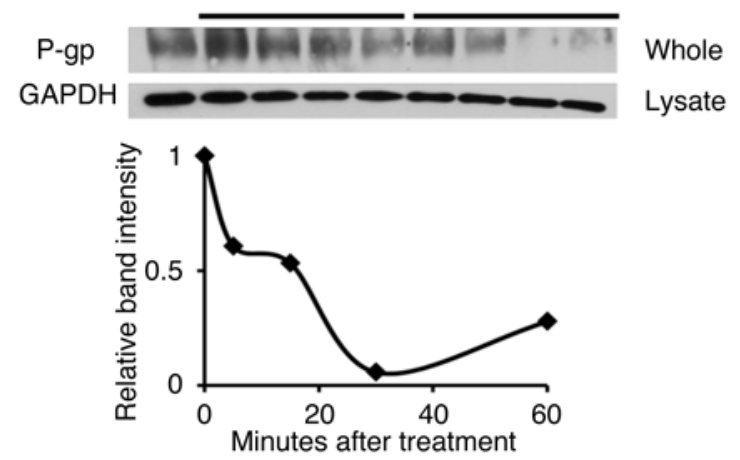

D Non Control LEX

MMP-9

GAPDH
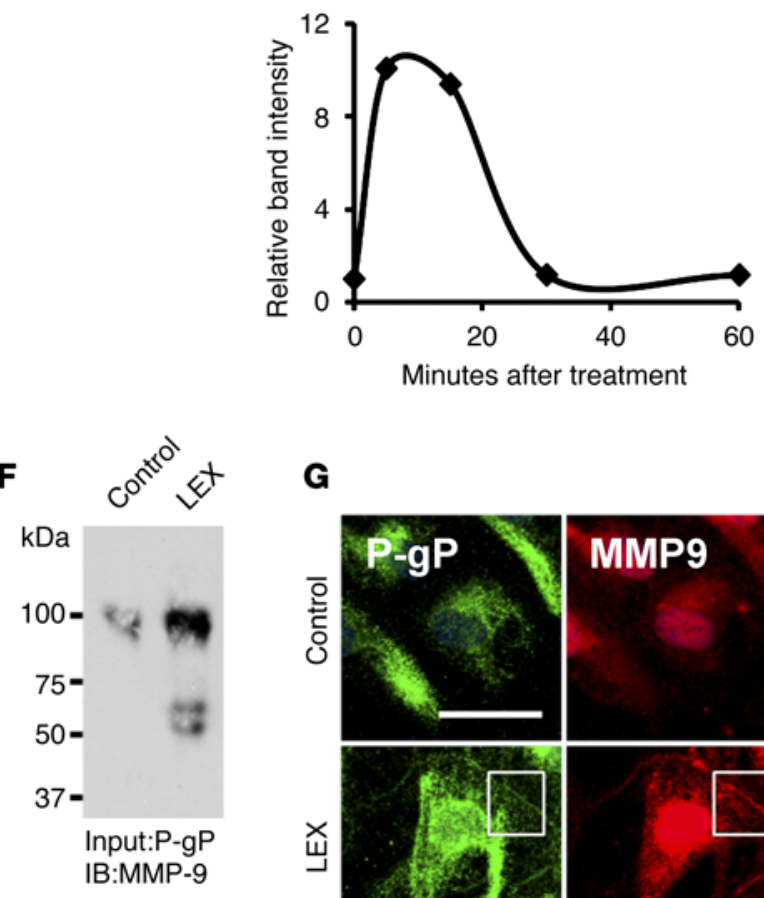

H
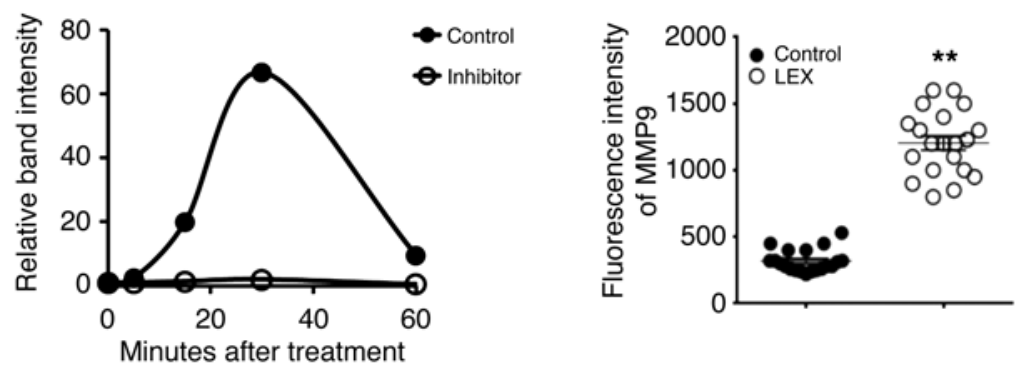

G
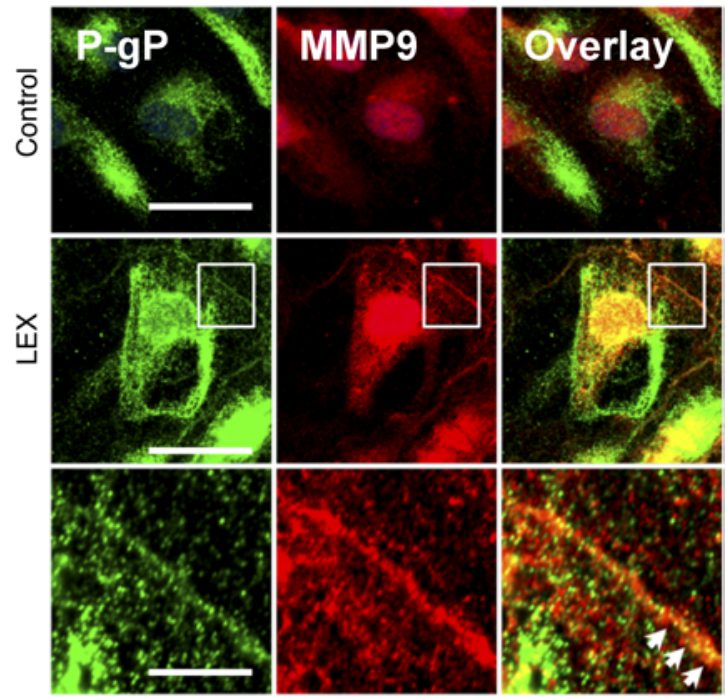
Figure 4. P-gp downmodulation by Lexiscan is mediated by MMPg cleavage and translocation to insoluble cytoskeletal fractions. (A) Schematic diagram of experimental procedure for analysis of Lexiscan's effect on P-gp solubility and excretion in primary brain endothelial cells (HBMVEC). (B and C) Western blot analysis of P-gp expression of HBMVEC upon activation with Lexiscan at different time points after lysis with RIPA buffer (B) or CSK buffer to measure P-gp levels in the cytoskeletal fraction (C). Band intensity was normalized to GAPDH and graphed. (D) Western blot analysis of MMPg in HBMVEC upon activation with Lexiscan at different time points. Band intensity was normalized using GAPDH and plotted. (E) Western blot analysis of secreted P-gp in HBMVEC upon activation with Lexiscan in absence or presence of an MMPg inhibitor: both short and long exposures of Western blot from MMPg inhibitor-treated samples are shown. Band intensity was plotted as graph. (F-H) Immunoprecipitation assay (F) of MMP9 with lysate from HBMVEC treated with vehicle (control) or $1 \mu \mathrm{M}$ of Lexiscan for 5 minutes. P-gp was pulled down using anti-P-gp antibody and immunoblotted with an anti-MMPg antibody. IFA (C) of MMP9 on HBMVEC treated with control (top panel) or $1 \mu \mathrm{M}$ of Lexiscan (middle panel) for 5 minutes. Cells were stained with P-gp (green) and MMPg (red). Nucleus was counterstained with DAPI (blue). Boxed region (inset) of Lexiscan-treated sample was magnified and displayed separately (bottom panel). Cell-surface colocalization of P-gp and MMPg is indicated by arrows. Scale bars: $25 \mu \mathrm{m}$ (upper and middle panels); 5 $\mu \mathrm{m}$ (bottom panels). (H) Analysis of intensity of fluorescence of MMPg from G. Fluorescent intensity of MMP9 was quantified and plotted as graph ( $n=$ 20). ${ }^{* *} P<0.01$ (2-tailed Student's $t$ test, 1 representative result of 3 different experiments).

6, A-C). This indicates that activation of A2A AR can alter BCRP expression/function. It is notable that Lexiscan's effect on P-gp occurred within 5 minutes compared with 15 minutes for BCRP. This suggests that A2A AR signaling effects on BCRP1 may be less sensitive compared with its effects on P-gp. To test whether these changes in P-gp expression in WT mice directly affect the functionality of P-gp and its substrate accumulation in the brain, we used the autofluorescent chemotherapeutic drug epirubicin, which allowed us to map its location and quantify its accumulation in the brain by fluorometry. Consistent with P-gp downmodulation, epirubicin accumulation in the brains of Lexiscantreated mice began to increase at 5 minutes; this was maintained up to 30 minutes and returned to baseline by 60 minutes (Figure 7C). This kinetic profile of P-gp downmodulation (Supplemental Figure 6, A-C) matched exactly with the kinetics of epirubicin accumulation in the brain (Figure 7C). Despite obtaining statistically significant epirubicin values in Lexiscan-treated mice, these values did not appear to match the fluorescent intensity of epirubicin observed in the brains of Lexiscan-treated mice (Figure 7D). Using a scanning scope, we captured the entire brain of mice treated with epirubicin with or without Lexiscan to visualize where epirubicin is accumulated in the brain and to quantify where it is localized (Figure 7D). Lexiscan treatment increased the accumulation of epirubicin prominently in the cerebral cortex, the cerebellum, and brain stem, and, to a lesser degree, in the olfactory bulb and hippocampus (Figure 7D). We next quantified the fluorescent intensity of specific areas in the brain to be compared with controls. We observed significant epirubicin intensity in the cerebral cortex, the cerebellum, and the hippocampus in Lexiscan-treated mice compared with control mice that received vehicle and epirubicin (Figure 7, D and E). This indicates that substantially more epirubicin accumulated in brain tissue of Lexiscan-treated mice than what we are able to extract (Figure 7C). To assess the inhibitory capacity of Lexiscan on P-gp in BBB permeability, we compared it to that of a well-known functional inhibitor of P-gp, PSC833, by measuring the intensity of epirubicin accumulation in the brain after PSC833 or Lexiscan treatment. We observed significantly lower epirubicin intensity in the brains of mice treated with PSC833 compared with Lexiscan-treated mice (Figure 7, D and E). Moreover, the PSC833 treatment group exhibited lower epirubicin intensity than even the control treatment group (Figure 7, D and E). This suggests that Lexiscan is a more potent inhibitor of P-gp than PSC833 (28). Based on these observations, we propose that $\mathrm{A} 2 \mathrm{~A} A \mathrm{AR}$ activation by Lexiscan rapidly and potently decreased P-gp expression and function, resulting in the accumulation of a P-gp substrate (epirubicin) in the brain .

We examined the effect of NECA treatment on BCRP expression compared with its effects on P-gp in brains of WT mice. NECA induced a gradual decrease in P-gp expression level, beginning at 2 hours; this was maintained for up to 18 hours, whereas the expression level of BCRP was not significantly affected (Figure 8, A-C). We examined the effect of NECA on P-gp downmodulation in BBB permeability to entry and accumulation of epirubicin in the brain. NECA-treated animals showed epirubicin accumulation in the brain that matched the kinetics of P-gp decrease in endothelial cells in the brain (Figure 8, B and D). Interestingly, the kinetics of epirubicin accumulation in the brain induced by Lexiscan and NECA were very similar to the time window of P-gp downmodulation in human primary brain endothelial cells in vitro (Figure 2D and Supplemental Figure 3D). Based on these findings, we conclude that signaling via the A2A AR on brain endothelial cells increases the transcellular pathway mediated by P-gp (summarized in Figure 9). We believe that we have provided a compelling case for adenosine signaling via the A2A receptor in P-gp regulation that in the future may lead us closer to drug delivery to the CNS.

\section{Discussion}

The $\mathrm{BBB}$ is necessary to protect the brain and maintain its homeostasis $(1,5,14)$. However, its restrictive nature hampers the ability to get therapeutics into the brain $(5,14)$. As the world population lives longer, the trend in neurodegenerative diseases increases, especially $\mathrm{AD}$ (14). Billions of dollars are spent on drug development to bypass the BBB or to modify drugs such that they would have easier access in traversing the $\mathrm{BBB}$, which blocks the delivery of the vast majority of drugs to the brain. After millions to billions of dollars are spent on developing these drugs, many of them are dropped from the pipeline, as they do not show efficacy or are too large to pass the BBB.

Cells and soluble molecules enter the brain through paracellular or transcellular pathways that are mediated by cell-to-cell junction or transporters, respectively $(1,14)$. A variety of transporters and receptors are highly expressed on brain endothelial cells that selectively restrict or allow the entry of substances, some of which are necessary for normal brain function, such as glucose and amino acids, while others are expelled $(14,55)$. Our previous studies showed that activation of the A2A AR increases BBB permeability to entry of large molecules that is mediated by increased paracellular permeability, induced RhoA activity, and rearrangement of the actin-cytoskeleton in brain endothelial cells $(44,59)$. However, 


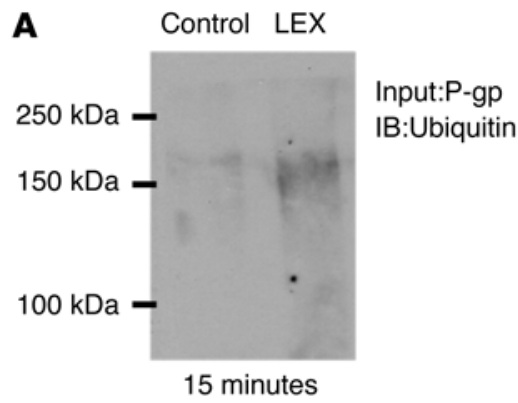

Summary of P-gp down-modulation by A2A AR signaling
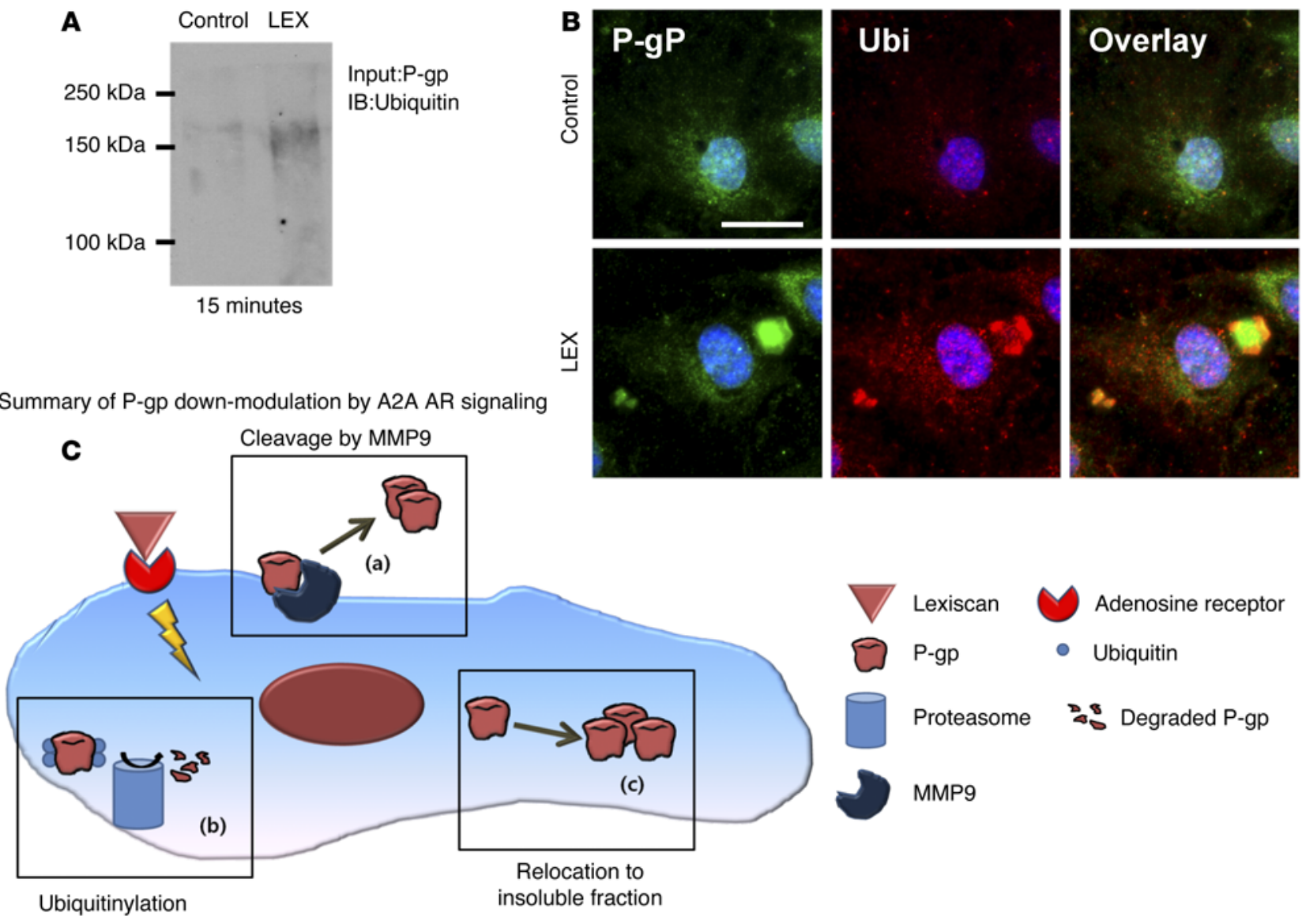

P-gp

Ubiquitin

Proteasome

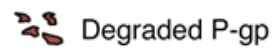

MMP9

Figure 5. P-gp downmodulation by Lexiscan is also mediated by ubiquitination. (A) Immunoprecipitation analysis of ubiquitination of P-gp in human primary brain endothelial cells upon activation with Lexiscan for 15 minutes. P-gp was pulled down with an anti-P-gp antibody and immunoblotted with an antiubiquitin antibody. (B) IFA depicting ubiquitination of P-gp in human primary brain endothelial cells upon activation with Lexiscan for 15 minutes. Cells were fixed, permeabilized, and stained with an anti-P-gp (green) and antiubiquitin (red) antibody. Nucleus was counterstained with DAPI (blue). Scale bar: $25 \mu \mathrm{m}$. (C) Schematic diagram depicting potential mechanisms of P-gp downregulation by Lexiscan. Upon activation of A2A AR, P-gp is cleaved by MMPg and secreted to the extracellular space (a), or ubiquitylated and digested by proteasome (b), or can relocate from soluble fraction to the insoluble fraction (c). These mechanisms may occur independently or in combination.

since molecules enter the CNS by transcellular and/or paracellular routes and the transcellular pathway is regulated by efflux transporters, such as P-gp, we wished to determine whether AR signaling also exerts regulatory effects on the transcellular pathway of the BBB mediated by P-gp $(5,11,17)$. Not surprisingly, many drugs are expelled by P-gp even before entering the brain and therefore are dropped from the drug pipeline in the course of their development (15). This poses tremendous economic loss and obstacles for public health, in particular, for treatment of CNS diseases $(5,11$, 22). Hence, it is imperative and urgent that we better understand how these processes operate.

In this study, we used a human brain endothelial cell line and primary human and mouse brain endothelial cells as in vitro models to investigate the impact of AR signaling on P-gp function, and then we determined whether our in vitro data can be recapitulated in vivo in mice. Our in vitro data showed that activation of AR significantly and potently altered P-gp expression/function. P-gp expression was rapidly downmodulated in both primary human and mouse brain endothelial cells and in a human brain endothelial cell line by activation of A2A AR with Lexiscan treatment.
In NECA treatment, downmodulation of P-gp occurred later than that of Lexiscan. We believe the difference in both Lexiscan and NECA's effects is a result of the difference in their halflives: Lexiscan's half-life is approximately 2.5 minutes, whereas NECA's is 5 hours. The downmodulation of P-gp by AR activation strikingly correlates with P-gp function that was confirmed by Rho123 accumulation and extravasation assays in primary human brain endothelial cells.

As we observed that a potent and rapid downmodulation of P-gp occurred over multiple time points after Lexiscan treatment of primary human brain endothelial cells, we hypothesized that AR activation may regulate P-gp expression and function by multiple mechanisms. We next investigated the molecular mechanism behind the rapid downmodulation of P-gp. In a cytoskeletal fractionation assay, we observed that a significant amount of P-gp is found in the insoluble fraction upon Lexiscan treatment of primary human brain endothelial cells. Moreover, we observed secreted P-gp in brain endothelial cell culture supernatants, suggesting P-gp is cleaved and released into the extracellular environment. Consistent with this notion, 
A
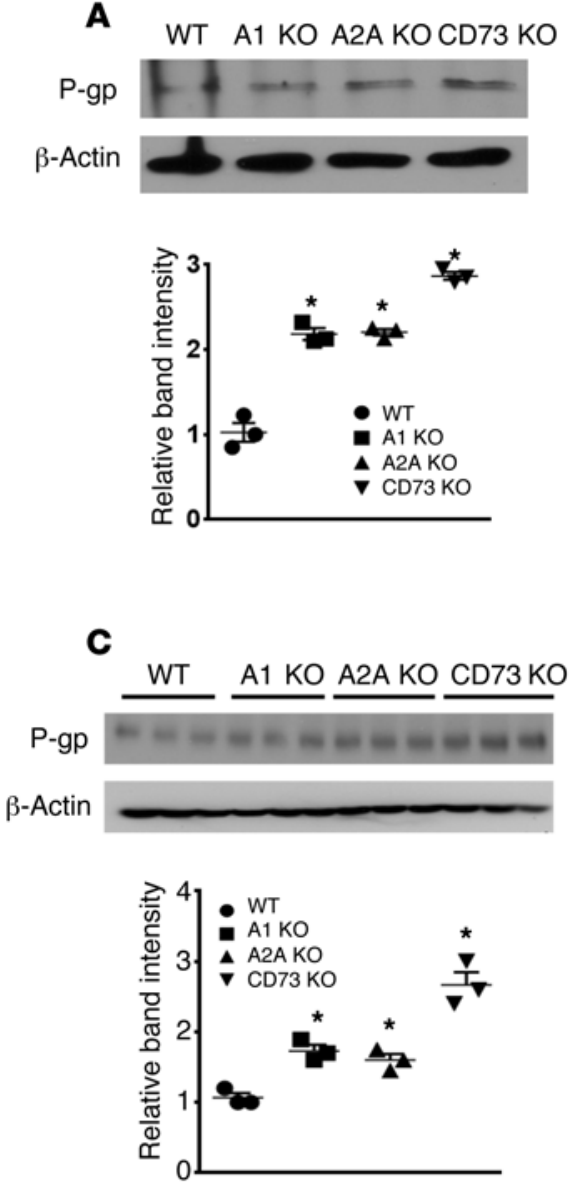

B

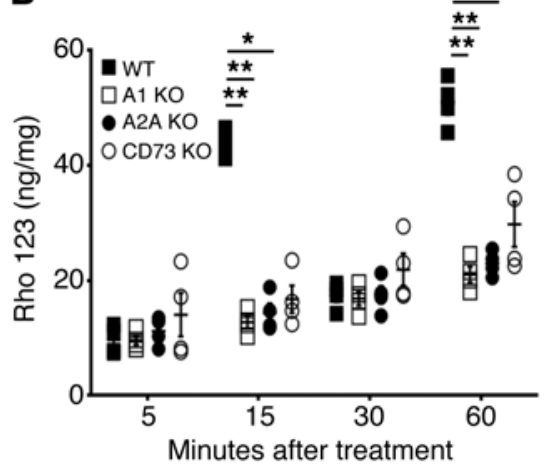

WT
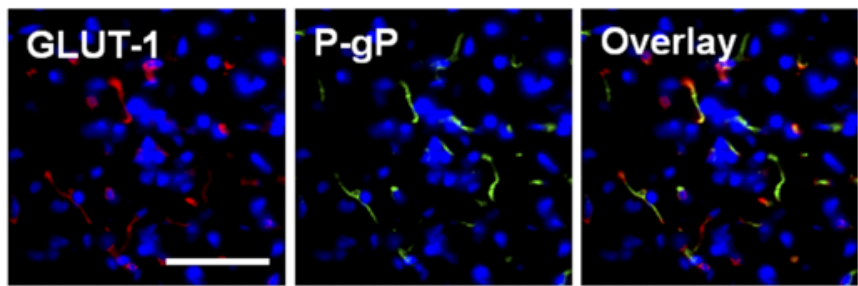

A1KO
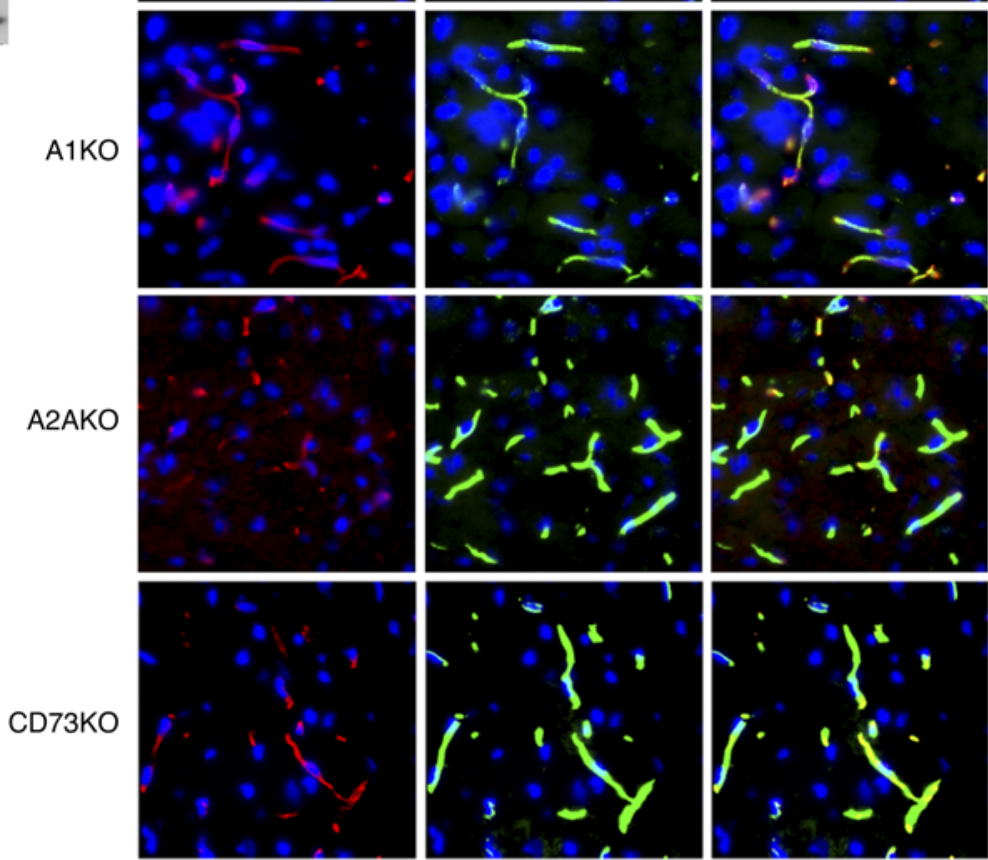

Figure 6. Ablation of CD73 or ARs increased P-gp expression and decreased P-gp substrate accumulation in the brain. (A) Western blot analysis of P-gp from primary brain endothelial cells of brains of WT, A1, A2A, and CD73 KO mice. $\beta$-Actin was used as a loading control. Intensity of bands was normalized with that of $\beta$-actin. ${ }^{*} P<0.05$ ( $n=3$ from 3 different Western blots, 2-tailed Student's $t$ test). (B) Rho123 uptake assay was performed using primary brain endothelial cells from brains of WT, A1, A2A, and CD73 KO mice. Cells were grown to confluence and treated with $2.5 \mu \mathrm{M}$ of Rho123 at 5, 15, 30, and 60 minutes. Cells were lysed with lysis buffer and were analyzed by fluorometry, with excitation at $488 \mathrm{~nm}$ and emission at $523 \mathrm{~nm}$. ${ }^{*} P<0.05 ;{ }^{*} P<0.01(n=4$, 2 -tailed Student's $t$ test, 1 representative result of 2 different experiments). (C) Western blot analysis of P-gp expression levels from brains of WT, A1 KO, A2A KO, and CD73 KO mice. $\beta$-Actin was used as loading control. Band intensity was normalized to that of $\beta$-actin and graphed. ${ }^{*} P<0.05(n=3,2$-tailed Student's $t$ test). (D) IFA on the brain of WT, A1 KO, A2A KO, and CD73 KO mice. Frozen brain sections were stained with GLUT1 (an endothelial marker, red) and P-gp (green) and counterstained with DAPI (blue). Nucleus was counterstained with DAPI (blue). Scale bar: $100 \mu \mathrm{m}$.

we found a concomitant increase in MMP9 levels that suggests that MMP9 may also be involved in downmodulation of P-gp upon AR activation. Moreover, we also determined that interaction between MMP9 and P-gp increases upon AR activation by immunoprecipitation assay and IFA, suggesting the cleavage of P-gp by MMP9 induces secretion of P-gp into the extracellular space. It is also reported that P-gp is regulated by ubiquitination
(64). In support of this, we observed rapid ubiquitinylation of P-gp by Lexiscan both by IFA and immunoprecipitation analysis. Together, these findings suggest that A2A AR-mediated regulation of P-gp expression/function occurs by these mechanisms independently or in combination.

Consistent with our in vitro findings, we observed AR activation exerted similar effects on P-gp expression and function 

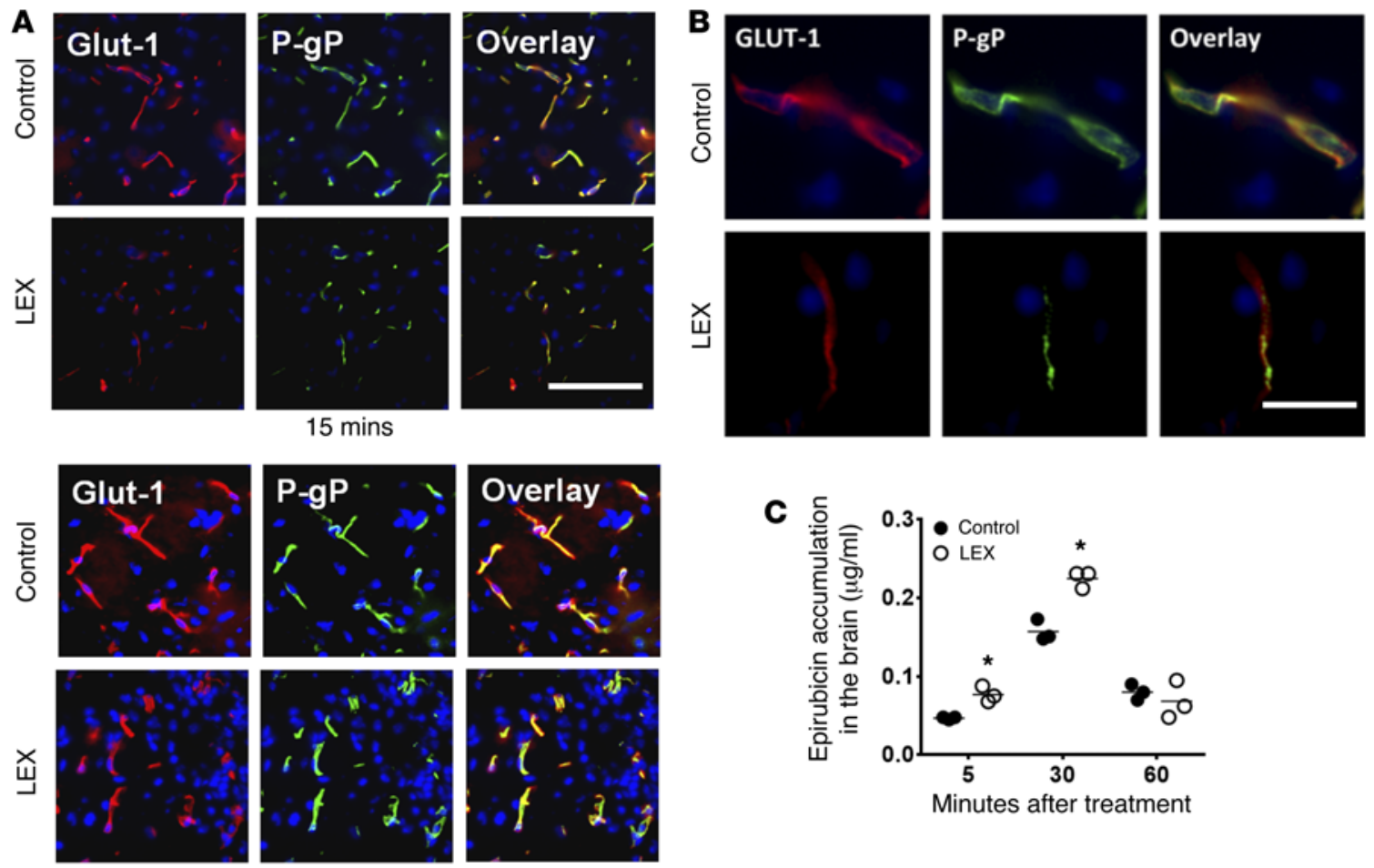

30 mins
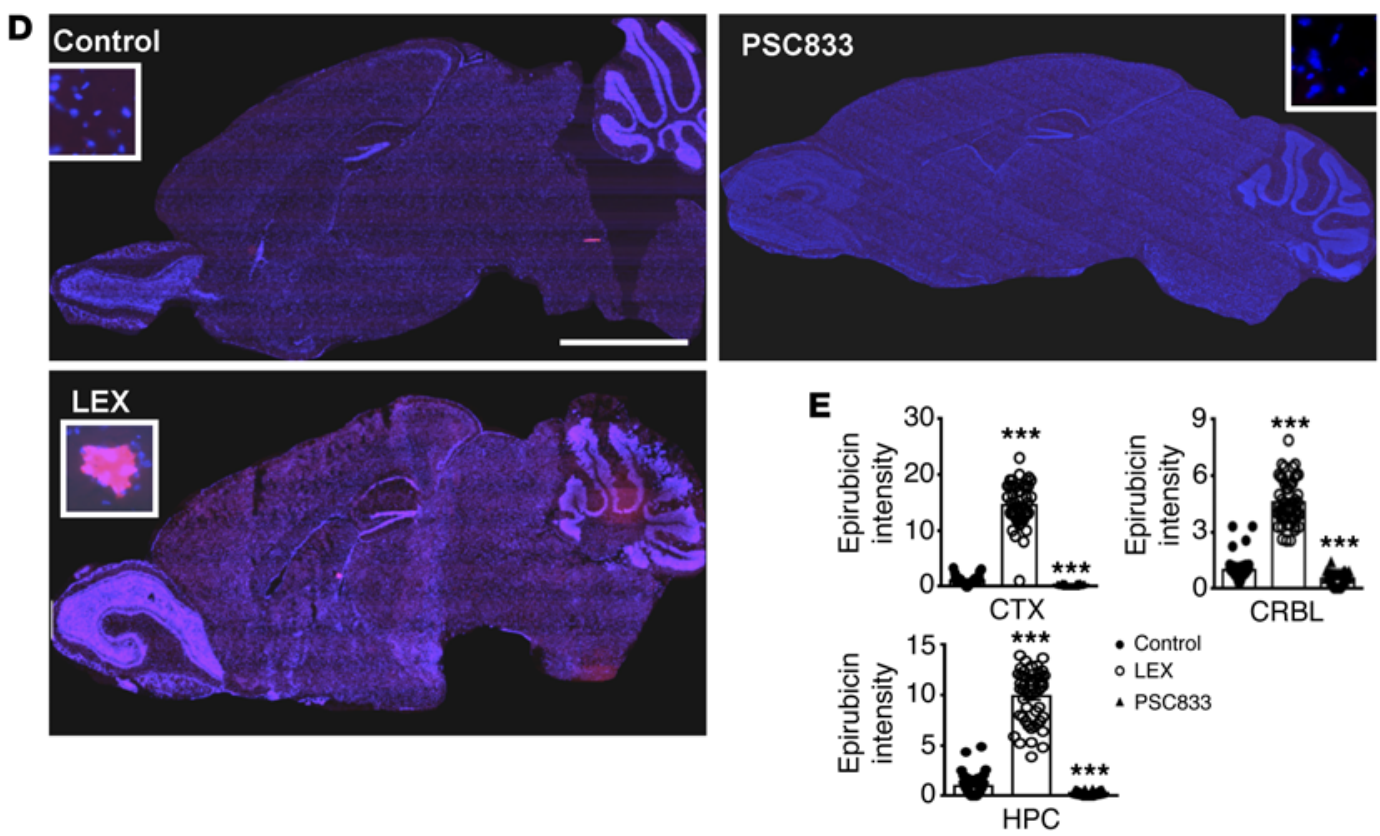

Figure 7. A2A receptor activation by Lexiscan induces rapid and reversible downmodulation of P-gp expression and function in brain vascular endothelial cells in WT mice. (A) Immunofluorescence images of brains from Lexiscan-treated mice of WT brain at 15 and 30 minutes after Lexiscan treatment. Frozen brain sections were stained with GLUT1 (red), or P-gp (green) and counterstained with DAPI (blue). (B) Enlarged image of A. (C) Brain epirubicin accumulation assay in the Lexiscan- and vehicle-treated (control) mice. $10 \mathrm{mg} / \mathrm{kg}$ of epirubicin was injected intravenously with or without $0.05 \mathrm{mg} / \mathrm{kg}$ of Lexiscan. Mice were perfused with ice-cold PBS and sacrificed at different time points. The accumulation of epirubicin in the brain was measured using fluorometric excitation at $488 \mathrm{~nm}$ and emission at $590 \mathrm{~nm} .{ }^{*} P<0.05(n=4$, 2-tailed Student's $t$ test). (D) Fluorescent microscopic analysis of epirubicin accumulation in the brains of mice treated with Lexiscan or PSC833 (a functional P-gp inhibitor) compared with vehicle (control). $10 \mathrm{mg} / \mathrm{kg}$ of epirubicin was injected intravenously with or without $0.05 \mathrm{mg} / \mathrm{kg}$ of Lexiscan or $50 \mathrm{mg} / \mathrm{kg}$ of PSC833 for 15 minutes. Mice were perfused with ice-cold PBS and sacrificed. Brain was sectioned for microscopic analysis for full-brain image, and focal zoomed image from cortex was laid as an inset. Epirubicin is in red, and nucleus was counterstained with DAPI (blue). (E) Intensity of epirubicin from different regions of cortex (CTX), cerebellum (CRBL), and hippocampus (HPC) from brains of control, Lexiscan-, or PSC833-injected animals was quantified and depicted as graphs. Scale bars: $100 \mu \mathrm{m}$ (A); $50 \mu \mathrm{m}$ (B) $5 \mathrm{~mm}$ (D). ${ }^{* * *} P<0.001(n=50$, 2-tailed Student's $t$ test). 
A

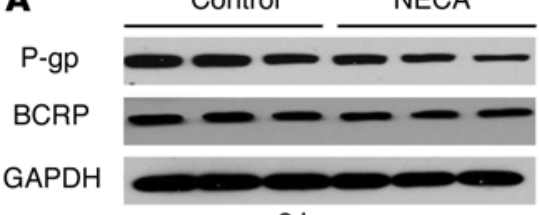

2 hours

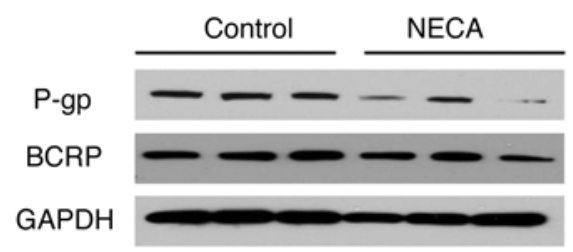

18 hours

C
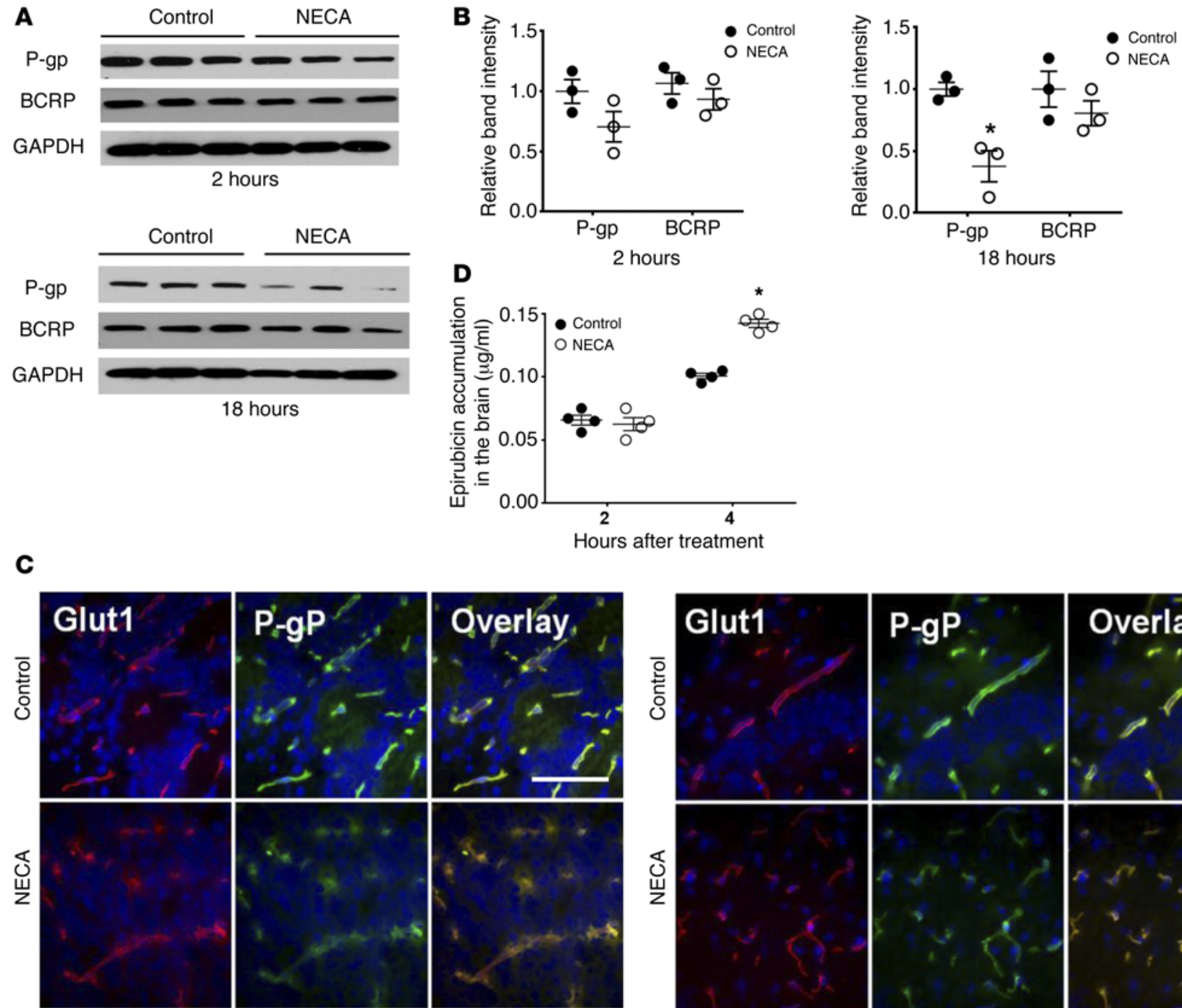

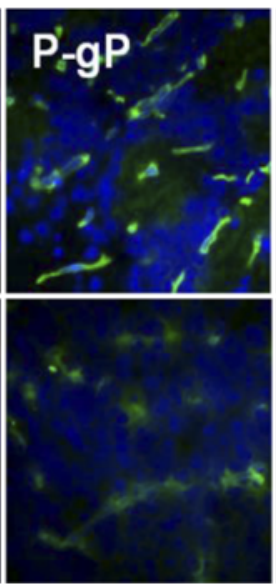

2 hours
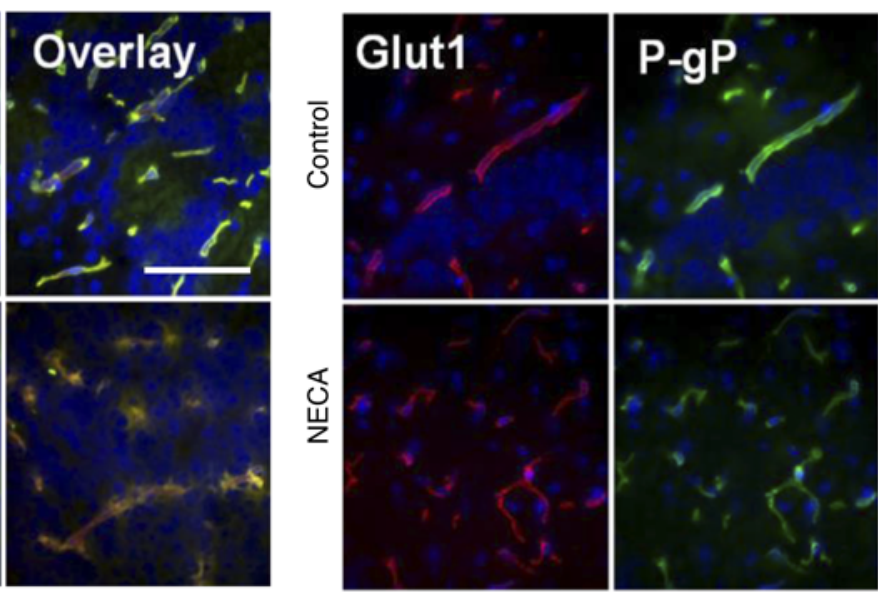

18 hours

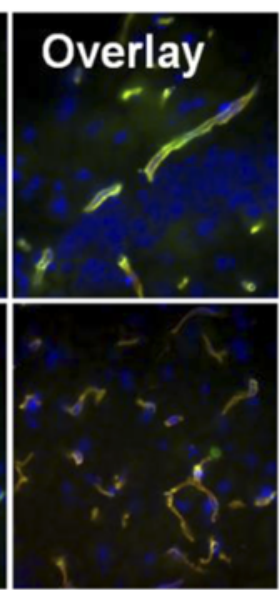

Figure 8. Broad-spectrum AR agonist NECA induces gradual and delayed downmodulation of P-gp expression and function in brain vascular endothelial cells in WT mice. (A) Western blot analysis of P-gp and BCRP1 from mouse brain at 2 and 18 hours after NECA treatment. (B) Enumeration of expression intensity of P-gp (top graph) or BCRP1 (bottom graph) bands from NECA treatment from Western blot analysis. Intensity of bands from NECA treatment group was divided by that of DMSO control. Acquired values were normalized by GAPDH and graphed. ${ }^{*} P<0.05$ ( $n=3$, 2-tailed Student's $t$ test). (C) IFA of P-gp in NECA-treated mouse brain at 2 and 18 hours after treatment. For IFA, brain frozen section were stained with GLUT1 (red) or P-gp (green) and counterstained with DAPI (blue). Scale bar: $100 \mu \mathrm{m}$. (D) Epirubicin brain accumulation assays in NECA-treated mice were performed after $0.08 \mathrm{mg} / \mathrm{kg}$ of NECA was injected intravenously for indicated time and, subsequently, $10 \mathrm{mg} / \mathrm{kg}$ of epirubicin was intravenously injected. At 15 minutes after epirubicin treatment, mice were perfused with ice-cold PBS and sacrificed at different time points. The accumulation of epirubicin in the brain was measured using fluorometry, with excitation at $488 \mathrm{~nm}$ and emission at $590 \mathrm{~nm}$. ${ }^{*} P<0.05$ ( $n=4,2$-tailed Student's $t$ test).

in vivo. In mouse brain endothelial cells, Lexiscan's effects on P-gp was rapid, occurring within 5 minutes, whereas the effect of NECA was observed 2 hours later. We also determined that A2A AR activation exerted its effects on BCRP1 expression/ function as potently as it did on P-gp. This suggests that both P-gp and BCRP1, which have been previously shown to function cooperatively, are regulated by A2A AR. A noted difference is that Lexiscan's effects on BCRP1 occurred 10 minutes later than its effects on P-gp. Thus, it is possible that P-gp activity may be more sensitive to A2A AR regulation than that of BCRP1. However, a more thorough study of BCRP1 regulation by A2A AR would be required to make this determination. As proof of prin- ciple that AR activation causes P-gp downmodulation, resulting in increased accumulation of P-gp substrates, we examined the effects of Lexiscan treatment on the accumulation of the chemotherapeutic drug epirubicin, which is a P-gp substrate. We observed that AR activation increased the accumulation of epirubicin in the brain that coincides with the kinetics of P-gp downmodulation. Consistent with our observation in vitro in human brain endothelial cells, Lexiscan's effect on accumulation of epirubicin was rapid, whereas NECA's was gradual. We believe this difference in permeability kinetics between the 2 agonists stems from differences in their half-lives (Lexiscan, 2.5 minutes; NECA, 5 hours). 
(a)
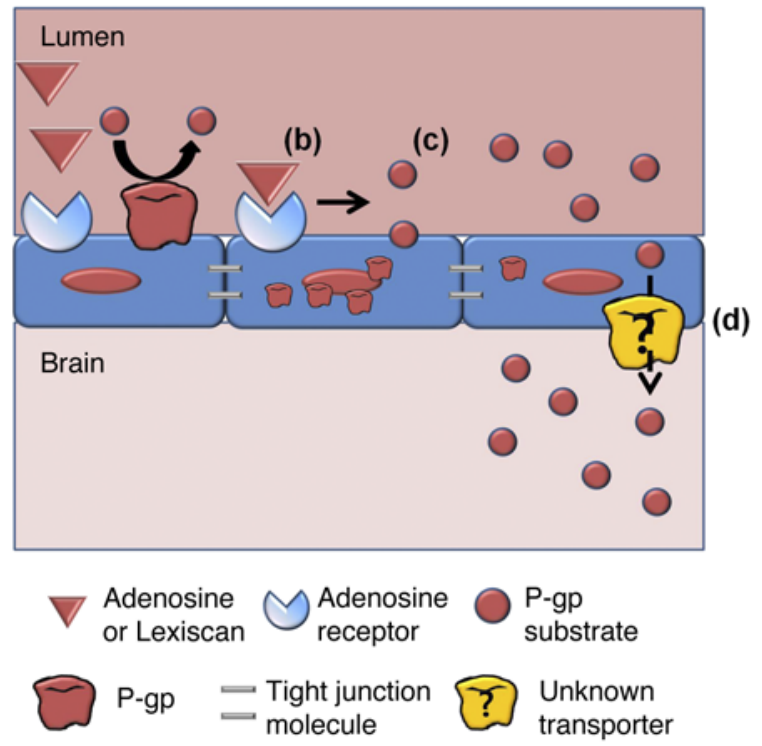

P-gp has long posed a tremendous hindrance to drug delivery to the brain and across the biological barrier in general $(9,22)$. This molecule functions by expelling drugs and xenobiotics from cells, and it alters drug pharmacokinetics (26). Its broad substrate spectrum allows it to expel major classes of drugs $(26,65)$. Moreover, P-gp expression or upregulation in various cancers and cell types poses a poor prognosis for cancers and cancer treatment (17). Therefore, our data showing that signaling via the A2A AR alters P-gp function have very broad appeal beyond the CNS. These data suggest that AR modulation may be a bona fide mechanism of altering P-gp function to effectively treat major cancers in general. We propose that these studies stand to open the door to studies well beyond modulation of the BBB.

Until recently, the brain was considered a formidable fortress that doesn't allow the entry of molecules or cells into the CNS. However, technological advancement and emerging studies have revealed that the brain is not totally cut off from the rest of the body; rather, it is selectively separated in order to maintain proper brain physiology $(1,5)$. Adenosine is a damage/ danger-signaling molecule that responds to cell stress or tissue damage by inducing a cascade of events involving recruitment of cells and substances across biological barriers needed to repair damaged tissues $(46,48)$. Therefore, adenosine is an endogenous (built in) modulator that regulates BBB permeability to recruit molecules into the CNS (to repair it) during CNS damage or stress. We posit that this system operated by AR signaling functions as a door and that adenosine is the key that signals its opening. We propose that this built-in mechanism relies on the extremely short half-life of adenosine (about 10 seconds) to reverse $\mathrm{BBB}$ permeability.

We believe that in the future, A2A AR modulation of the BBB may offer a safe means of delivering drugs into the CNS. AR modulation of BBB permeability provides a kinetic window of P-gp downmodulation that can be exploited to deliver therapeutics to treat diseases ranging from primary brain tumors to AD. It offers a time line of drug delivery to the brain that can be transient or gradual, depending on the A2A AR agonist used.
Figure 9. Mechanism of regulation of transcellular permeability by A2A AR signaling. (a) In basal status, single layer of brain endothelial cells highly express transporters including P-gp. (b) When AR is activated by adenosine or Lexiscan, (c) it downregulates P-gp on brain endothelial cells (d), which increases transcellular permeability and delivery of P-gp substrate into the brain. When P-gp substrate crosses the brain endothelial cells, it is still not clear which transporters subsequently aid the final delivery of molecules into the brain side.

Importantly, BBB permeability is reversed, returning to steady state after the effects of the agonist wane. ARs and the enzymes that generate extracellular adenosine are expressed directly on BBB cells, and some AR pharmacological agents, including Lexiscan, are FDA approved and may lessen some potential hurdles for use in humans. In summary, our data showing that AR mediates P-gp function in BBB permeability are exciting, highly translational, and stand to have a high impact on public health. In the future, A2A AR modulation of BBB via regulation of P-gp may provide a real alternative in treating brain tumors such as gliomas, which are incurable and have an average survival time of 18 months.

\section{Methods}

Cells and materials. Human brain endothelial cell (HCMEC-D3) was provided by Barbette Weksler (Weill Cornell Medical Center). Primary human brain endothelial cells were purchased from Cell Systems. Lexiscan was purchased from Toronto Research Chemicals. Rho123 was purchased from Sigma-Aldrich. Epirubicin (catalog 3260), PSC833 (catalog 4042), and NECA (catalog 1691) were purchased from Tocris. Anti-human CD31 antibody (catalog FAB3567C) was purchased from R\&D Bioscience. P-gp antibodies were purchased as follows: catalog GTX108354, GTX23364 from GeneTex; catalog 557001 from BD Biosciences; catalog EPR10364-57 from Abcam. ABCG2 (catalog GTX23380) and GLUT1 (catalog GTX100684) antibodies were purchased from GeneTex. Anti-MMP9 antibody (catalog ab38898) was purchased from Abcam. Alexa Fluor 594conjugated wheat germ agglutinin (WGA) (catalog W11262, Thermo Fisher) was provided by Marci Scidmore (Cornell University). Mouse anti-caveolin-1 antibody (catalog 610493, BD Biosciences) was provided by Gary Whittaker (Cornell University).

Mouse primary brain endothelial cell culture. Mice were sacrificed under the IACUC protocol of the College of Veterinary Medicine of Cornell University. Meninges of brain were removed and ground using a plunger and centrifuged at 3,000 $g$ for 5 minutes. The obtained pellet was dissolved in $18 \%$ dextran and centrifuged at 10,000 $\mathrm{g}$ for 10 minutes and digested with DMEM containing collagenase, DNAse, and dispase at $37^{\circ} \mathrm{C}$ for 75 minutes. The sample was centrifuged for 5 minutes at $3,200 \mathrm{~g}$, and the pellet was washed with warm PBS. The pellet was resuspended in DMEM/F12 with puromycin, heparin, $20 \%$ PDS, and L-glutamine and plated onto the proper plate.

Subcellular localization analysis of P-gp in brain endothelial cells. To analyze the subcellular localization of P-gp in the HCMEC-D3 and primary brain endothelial cells, we plated cells on coverslips and fixed them with PFA for 20 minutes. Cells were washed with $0.5 \%$ BSA 2 times and incubated with $5 \%$ goat serum for 45 minutes. Cells were incubated with 1:200 anti P-gp (BD Biosciences) overnight. Coverslips were washed 2 times and incubated with anti-mouse secondary 
antibody conjugated with Alexa Fluor 647 for 1 hour. Cell surface was stained with Alexa Fluor 568 WGA (Invitrogen, 1:200) or antiCD31 (R\&D Bioscience, 1:200). For costaining of caveolae, cells were stained with mouse anti-caveolin-1 (BD Biosciences, 1:200)

Immunoprecipitation assay. 4G Sepharose beads were purchased from Invitrogen and incubated with human P-gp antibody (BD Biosciences) overnight at $4^{\circ} \mathrm{C}$ using a rocking shaker. Beads were washed twice using lysis buffer containing protease inhibitor, and lysates from primary brain endothelial cells were incubated overnight at $4^{\circ} \mathrm{C}$ using a rocking shaker. Samples were spun down at 4,000 $g$ for 2 minutes and washed with lysis buffer containing protease inhibitor 3 times. Samples were eluted with $0.1 \mathrm{M}$ glycine ( $\mathrm{pH} 2.8$ ), and the eluent was mixed with sample buffer, which was loaded on a 10\% SDS-PAGE gel run for 2 hours at $100 \mathrm{~V}$ and subsequently transferred to nitrocellulose paper for Western blot analysis.

Western blot. HCMEC-D3 and HBMVEC cells were plated in 12 -well plates and were grown until they reached $100 \%$ confluency. The media was replaced with fresh media containing $1 \mu \mathrm{M}$ of Lexiscan or NECA with proper vehicle control (DMSO) for up to 72 hours. Cells were lysed by lysis buffer (RPMI) containing protease inhibitor cocktail and stored for later use $\left(-70^{\circ} \mathrm{C}\right)$. For cytoskeletal fraction analysis, cells were lysed in CSK buffer $(100 \mathrm{mM} \mathrm{NaCl}, 300 \mathrm{mM}$ sucrose, $3 \mathrm{mM} \mathrm{MgCl}$, $10 \mathrm{mM}$ PIPES, pH 6.8). For brain samples, half of the brain was homogenized and lysed with RPMI lysis buffer and centrifuged at 17,000 $g$ for 20 minutes and 1:10 diluted samples were used for analysis. Sample was loaded on 7\% SDS PAGE at $100 \mathrm{~V}$ for 1 hour and transferred to the nitrocellulose paper. It was blocked with $1 \%$ BSA and incubated with anti-P-gp antibody (Genetex, 1:2000) overnight. Subsequently, it was washed with TBST and incubated with anti-rabbit secondary antibody (1:2000) for 1 hour. It was washed with TBST and developed with ECL substrate and exposed to $\mathrm{x}$-ray film. Anti-GAPDH or $-\beta$-actin antibody was used as loading control. The intensity of band was analyzed with densitometric analysis and plotted as a graph for analysis of time-course effect of AR signaling on P-gp expression.

Rho123 uptake assay. Human brain endothelial cells and primary brain endothelial cells were plated onto 48 -well plates until 100\% confluence was reached, and media were replaced with fresh media containing Rho123 $(2.5 \mu \mathrm{M})$ with different concentrations of Lexiscan, NECA, or PSC833. Reaction was halted by adding $250 \mathrm{ul}$ of ice-cold PBS. Plate was washed with $250 \mu \mathrm{l}$ of ice-cold PBS 3 times and lysed with lysis buffer. Each sample was analyzed using Synergy Fluometer (Biotek), with excitation at $488 \mathrm{~nm}$ and emission at 523 $\mathrm{nm}$. Concentration of Rho123 was analyzed using standard curve created by serial dilution of Rho123. Final concentration of Rho123 was normalized using protein concentration of lysate measured using Bradford assay (Bio-Rad). For microscopic analysis of Rho123 uptake, cells were cultured on coverslip and Rho123 was treated with or without Lexiscan or NECA with DMSO as control. Reaction was terminated by adding ice-cold PBS and washed with ice-cold PBS 3 times. Cells were fixed with PFA and costained with anti-human P-gp. Cells were visualized with a Zeiss fluorescent microscope and captured with axiovision software.
Rho123 extravasation assay. Primary human brain endothelial cells were plated on $3 \mu \mathrm{m}$ of porous membrane (Corning) until 100\% confluency was reached. Media was replaced with HBSS and acclimated for 4 hours before initiation of experiments. Rho123 $(2.5 \mu \mathrm{M})$ with or without Lexiscan or NECA $(0.25 \mu \mathrm{M})$ was applied on the upper chamber of porous membrane for up to 48 hours. Media at the bottom chamber were collected at different time points, and each sample was analyzed using Synergy Fluorometer (Biotek), with excitation at 488 $\mathrm{nm}$ and emission at $523 \mathrm{~nm}$.

Epirubicin brain accumulation assay. For Lexiscan study, $10 \mathrm{mg} / \mathrm{kg}$ of epirubicin was injected with or without Lexiscan $(0.05 \mathrm{mg} / \mathrm{kg})$ at different time points, and mice were sacrificed. Mice were injected with NECA $(0.08 \mathrm{mg} / \mathrm{kg})$ or vehicle control at different time points, after which $10 \mathrm{mg} / \mathrm{kg}$ of epirubicin was injected intravenously for 15 minutes; mice were then sacrificed. At indicated time points, mice were perfused with ice-cold PBS and brain was collected for further analysis. Brain was ground and spun down in Tris- $\mathrm{HCl}(\mathrm{pH}=8.0)$ at 17,000 $\mathrm{g}$ for $30 \mathrm{~min}-$ utes, and supernatant was transferred to a new tube and precipitated with the same volume of $\mathrm{MeOH}$. Samples were spun down at 17,000 $\mathrm{g}$ for 30 minutes, and the concentration for epirubicin was analyzed with fluorimetry with emission at $488 \mathrm{~nm}$ and excitation at $575 \mathrm{~nm}$.

IFA of frozen section. Mice were treated with Lexiscan or NECA at different time points and were infused with ice-cold PBS and sacrificed. Half of the brain was cut and put in the cassette and filled with OCT solution. Samples were snap-frozen with liquid nitrogen, cut with cryostat $(10 \mu \mathrm{m})$ and fixed with acetone for 5 minutes, and incubated with C219 for P-gp stain (1:100) and Glut1 for endothelial cell marker $(1: 100)$ at $4^{\circ} \mathrm{C}$ overnight. Sections were washed with PBS and additionally stained with secondary antibody conjugated with fluorochrome. Full images of the brain sections were visualized and recorded using Aperio Scan Scope (Leica Biosystems).

Statistics. All statistical analysis was carried out using GraphPad 5.0 software. Statistical significance was assessed using unpaired 2-tailed Student's $t$ test. $P$ values of less than 0.05 were considered to be statistically significant.

Study approval. All animal work was done in accordance with Public Health Service guidelines and was approved by Cornell's Institutional Animal Care and Use Committee (protocol no. 2008-0092).

\section{Author contributions}

DGK and MSB designed experiments, performed experiments, analyzed data, and wrote the manuscript.

\section{Acknowledgments}

This study was supported by NIH grant R01 NS063011 (to M.S. Bynoe) and a grant from the Kwanjung Educational Foundation for Study Abroad (to D.G. Kim).

Address correspondence to: Margaret S. Bynoe, Cornell University College of Veterinary Medicine, C5 149 Veterinary Medical Center, Cornell University, Ithaca, New York 14853, USA. Phone: 607.253.4023; E-mail: msb76@cornell.edu.
1. Abbott NJ. Astrocyte-endothelial interactions and blood-brain barrier permeability. JAnat. 2002;200(6):629-638.

2. Abbott NJ, Ronnback L, Hansson E. Astrocyte- endothelial interactions at the blood-brain barrier. Nat Rev Neurosci. 2006;7(1):41-53.

3. Abbott NJ. Blood-brain barrier structure and function and the challenges for CNS drug delivery.J
Inherit Metab Dis. 2013;36(3):437-449.

4. Ballabh P, Braun A, Nedergaard M. The bloodbrain barrier: an overview: structure, regulation, and clinical implications. Neurobiol Dis. 
2004;16(1):1-13

5. Deeken JF, Loscher W. The blood-brain barrier and cancer: transporters, treatment, and Trojan horses. Clin Cancer Res. 2007;13(6):1663-1674.

6. Gabathuler R. Approaches to transport therapeutic drugs across the blood-brain barrier to treat brain diseases. Neurobiol Dis. 2010;37(1):48-57.

7. Neuwelt EA, Maravilla KR, Frenkel EP, Rapaport SI, Hill SA, Barnett PA. Osmotic blood-brain barrier disruption. Computerized tomographic monitoring of chemotherapeutic agent delivery. JClin Invest. 1979;64(2):684-688.

8. Elliott PJ, Hayward NJ, Dean RL, Blunt DG, Bartus RT. Intravenous RMP-7 selectively increases uptake of carboplatin into rat brain tumors. Cancer Res. 1996;56(17):3998-4005.

9. Pardridge WM. Drug transport across the blood-brain barrier. J Cereb Blood Flow Metab. 2012;32(11):1959-1972.

10. Cook AM, Mieure KD, Owen RD, Pesaturo AB, Hatton J. Intracerebroventricular administration of drugs. Pharmacotherapy. 2009;29(7):832-845.

11. Daneman R, Prat A. The blood-brain barrier. Cold Spring Harb Perspect Biol. 2015;7(1):a020412.

12. Abbott NJ. Astrocyte-endothelial interactions and blood-brain barrier permeability. J Anat. 2002;200(6):629-638.

13. Pascual JM, et al. GLUT1 deficiency and other glucose transporter diseases. Eur JEndocrinol. 2004;150(5):627-633.

14. Pardridge WM. The blood-brain barrier: bottleneck in brain drug development. NeuroRx. 2005;2(1):3-14.

15. Begley DJ. ABC transporters and the blood-brain barrier. Curr Pharm Des. 2004;10(12):1295-1312.

16. Beaulieu E, Demeule M, Ghitescu L, Beliveau R. P-glycoprotein is strongly expressed in the luminal membranes of the endothelium of blood vessels in the brain. Biochem J. 1997;326(pt 2):539-544.

17. Chung HC, et al. P-glycoprotein: the intermediate end point of drug response to induction chemotherapy in locally advanced breast cancer. Breast Cancer Res Treat. 1997;42(1):65-72.

18. Filipits M, Suchomel RW, Lechner K, Pirker R. Immunocytochemical detection of the multidrug resistance-associated protein and P-glycoprotein in acute myeloid leukemia: impact of antibodies, sample source and disease status. Leukemia. 1997;11(7):1073-1077.

19. Oda Y, Rose I, Radig K, Wagemann W, Mittler U, Roessner A. Expression of MDR1/p-glycoprotein and multidrug resistance-associated protein in childhood solid tumours. Virchows Arch. 1997;430(2):99-105.

20. Hebert MF. Contributions of hepatic and intestinal metabolism and P-glycoprotein to cyclosporine and tacrolimus oral drug delivery. Adv Drug Deliv Rev. 1997;27(2-3):201-214.

21. Kusuhara H, Suzuki H, Terasaki T, Kakee A, Lemaire M, Sugiyama Y. P-Glycoprotein mediates the efflux of quinidine across the blood-brain barrier. JPharmacol Exp Ther. 1997;283(2):574-580.

22. Schinkel AH. P-Glycoprotein, a gatekeeper in the blood-brain barrier. Adv Drug Deliv Rev. 1999;36(2-3):179-194.

23. Yang CP, DePinho SG, Greenberger LM, Arceci RJ, Horwitz SB. Progesterone interacts with
P-glycoprotein in multidrug-resistant cells and in the endometrium of gravid uterus. J Biol Chem. 1989;264(2):782-788.

24. Gottesman MM, Ling V. The molecular basis of multidrug resistance in cancer: the early years of P-glycoprotein research. FEBS Lett. 2006;580(4):998-1009.

25. Gottesman MM, Ambudkar SV, Xia D. Structure of a multidrug transporter. Nat Biotechnol. 2009;27(6):546-547.

26. Aller SG, et al. Structure of P-glycoprotein reveals a molecular basis for poly-specific drug binding. Science. 2009;323(5922):1718-1722.

27. Horton JK, et al. Characterization of a novel bisacridone and comparison with PSC 833 as a potent and poorly reversible modulator of P-glycoprotein. Mol Pharmacol.1997;52(6):948-957.

28. Mayer U, Wagenaar E, Dorobek B, Beijnen JH, Borst P, Schinkel AH. Full blockade of intestinal P-glycoprotein and extensive inhibition of blood-brain barrier P-glycoprotein by oral treatment of mice with PSC833. JClin Invest. 1997;100(10):2430-2436

29. Rao US, Fine RL, Scarborough GA. Antiestrogens and steroid hormones: Substrates of the human P-glycoprotein. Biochem Pharmacol. 1994;48(2):287-292.

30. Pawlik A, Gawrońska-Szklarz B, BaśkiewiczMasiuk M, Machaliński B, Safranow K. Involvement of P-glycoprotein in the release of cytokines from peripheral blood mononuclear cells treated with methotrexate and dexamethasone. JPharm Pharmacol. 2005;57(11):1421-1425.

31. Paterson JK, Gottesman MM. P-Glycoprotein is not present in mitochondrial membranes. Exp Cell Res. 2007;313(14):3100-3105.

32. Shen Y, Chu Y, Yang Y, Wang Z. Mitochondrial localization of P-glycoprotein in the human breast cancer cell line MCF-7/ADM and its functional characterization. Oncol Rep. 2012;27(5):1535-1540.

33. Yamagishi T, Sahni S, Sharp DM, Arvind A, Jansson PJ, Richardson DR. P-glycoprotein mediates drug resistance via a novel mechanism involving lysosomal sequestration. J Biol Chem. 2013;288(44):31761-31771.

34. Fu D, Roufogalis BD. Actin disruption inhibits endosomal traffic of P-glycoprotein-EGFP and resistance to daunorubicin accumulation. Am J Physiol Cell Physiol. 2007;292(4):C1543-C1552.

35. Staud F, Pavek P. Breast cancer resistance protein (BCRP/ABCG2). Int J Biochem Cell Biol. 2005;37(4):720-725.

36. Insel PA, Patel HH. Membrane rafts and caveolae in cardiovascular signaling. Curr Opin Nephrol Hypertens. 2009;18(1):50-56.

37. Bastiani M, Parton RG. Caveolae at a glance. JCell Sci. 2010;123(22):3831-3836.

38. Lajoie P, Nabi IR. Lipid rafts, caveolae, and their endocytosis. In: Kwang WJ, ed. International Review of Cell and Molecular Biology. Vol. 323. San Diego, CA: Academic Press; 2010:135-163.

39. Zhu M, Le Moyec L, Starzec A, Stierle V, Marbeuf-Gueye C. Caveolin-1 and doxorubicininduced P-glycoprotein modulate plasma cholesterol membrane accessibility in erythrolymphoblastic cell line. Anticancer Res. 2010;30(9):3451-3458.
40. Tome ME, et al. Identification of P-glycoprotein co-fractionating proteins and specific binding partners in rat brain microvessels. J Neurochem. 2015;134(2):200-210.

41. Noack A, et al. Drug-induced trafficking of p-glycoprotein in human brain capillary endothelial cells as demonstrated by exposure to mitomycin C. PLoS One. 2014;9(2):e88154.

42. Jette L, Potier M, Beliveau R. P-glycoprotein is a dimer in the kidney and brain capillary membranes: effect of cyclosporin A and SDZ-PSC833. Biochemistry. 1997;36(45):13929-13937.

43. van Asperen J, et al. Enhanced oral bioavailability of paclitaxel in mice treated with the P-glycoprotein blocker SDZ PSC833. Br J Cancer. 1997;76(9):1181-1183.

44. Kim DG, Bynoe MS. A2A adenosine receptor regulates the human blood-brain barrier permeability. Mol Neurobiol. 2014;52(1):664-678.

45. Carman AJ, Mills JH, Krenz A, Kim DG, Bynoe MS. Adenosine receptor signaling modulates permeability of the blood-brain barrier. J Neurosci. 2011;31(37):13272-13280.

46. Hasko G, Pacher P, Vizi ES, Illes P. Adenosine receptor signaling in the brain immune system. Trends Pharmacol Sci. 2005;26(10):511-516.

47. Hasko G, Linden J, Cronstein B, Pacher P. Adenosine receptors: therapeutic aspects for inflammatory and immune diseases. Nat Rev Drug Discov. 2008;7(9):759-770.

48. Jacobson KA, Gao ZG. Adenosine receptors as therapeutic targets. Nat Rev Drug Discov. 2006;5(3):247-264.

49. Fredholm BB, IJzerman AP, Jacobson KA, Linden J, Muller CE. International Union of Basic and Clinical Pharmacology. LXXXI. Nomenclature and classification of adenosine receptors - an update. Pharmacol Rev. 2011;63(1):1-34.

50. Fredholm BB, Arslan G, Halldner L, Kull B, Schulte G, Wasserman W. Structure and function of adenosine receptors and their genes. Naunyn Schmiedebergs Arch Pharmacol. 2000;362(4-5):364-374.

51. Mills JH, et al. CD73 is required for efficient entry of lymphocytes into the central nervous system during experimental autoimmune encephalomyelitis. Proc Natl Acad Sci US A. 2008;105(27):9325-9330.

52. Mills JH, Kim DG, Krenz A, Chen JF, Bynoe MS. A2A adenosine receptor signaling in lymphocytes and the central nervous system regulates inflammation during experimental autoimmune encephalomyelitis. J Immunol. 2012;188(11):5713-5722.

53. Bernas MJ, et al. Establishment of primary cultures of human brain microvascular endothelial cells to provide an in vitro cellular model of the blood-brain barrier. Nat Protocols. 2010;5(7):1265-1272.

54. Demeule M, Jodoin J, Gingras D, Béliveau R. $\mathrm{P}$-glycoprotein is localized in caveolae in resistant cells and in brain capillaries. FEBS Lett. 2000;466(2-3):219-224.

55. Jodoin J, et al. P-glycoprotein in blood-brain barrier endothelial cells: interaction and oligomerization with caveolins. J Neurochem. 2003;87(4):1010-1023.

56. Shvets E, Bitsikas V, Howard G, Hansen CG, 
Nichols BJ. Dynamic caveolae exclude bulk membrane proteins and are required for sorting of excess glycosphingolipids. Nat Commun. 2015;6:6867.

57. Mundy DI, Machleidt T, Ying Y-s, Anderson RGW, Bloom GS. Dual control of caveolar membrane traffic by microtubules and the actin cytoskeleton. J Cell Sci. 2002;115(22):4327-4339.

58. Lee JS, et al. Rhodamine efflux patterns predict P-glycoprotein substrates in the National Cancer Institute drug screen. Mol Pharmacol. 1994;46(4):627-638.

59. Carman AJ, Mills JH, Krenz A, Kim DG, Bynoe
MS. Adenosine receptor signaling modulates permeability of the blood-brain barrier. JNeurosci. 2011;31(37):13272-13280.

60. Mundell S, Kelly E. Adenosine receptor desensitization and trafficking. Biochim Biophys Acta. 2011;1808(5):1319-1328.

61. Lasley RD. Adenosine receptors and membrane microdomains. Biochim Biophys Acta. 2011;1808(5):1284-1289.

62. Velot $\mathrm{E}$, et al. Activation of the adenosine-A3 receptor stimulates matrix metalloproteinase-9 secretion by macrophages. Cardiovasc Res. 2008;80(2):246-254.
63. Chen H, Koupenova M, Yang D, Sume SS, Trackman PC, Ravid K. Regulation of MMP-9 expression by the $\mathrm{A} 2 \mathrm{~b}$ adenosine receptor and its dependency on TNF- $\alpha$ signaling. Exp Hematol. 2011;39(5):525-530.

64. Zhang Z, Wu JY, Hait WN, Yang JM. Regulation of the stability of P-glycoprotein by ubiquitination. Mol Pharmacol. 2004;66(3):395-403.

65. Kuo YC, Lu CH. Expression of P-glycoprotein and multidrug resistance-associated protein on human brain-microvascular endothelial cells with electromagnetic stimulation. Colloids Surf B Biointerfaces. 2012;91:57-62. 